\title{
The Effect of Economic Crises on Japan's Bilateral Trade Distribution: Examination of the Tohoku Earthquake Impact on African Markets
}

Onur BIYIK ( $\sim$ biyik-onur@fuji.waseda.jp )

Waseda University

\section{Research Article}

Keywords: Japanese international trade, panel data, gravity model, Tohoku earthquake, African markets

Posted Date: January 12th, 2022

DOI: https://doi.org/10.21203/rs.3.rs-1240372/v2

License: (c) (1) This work is licensed under a Creative Commons Attribution 4.0 International License.

Read Full License 


\title{
The Effect of Economic Crises on Japan's Bilateral Trade Distribution: Examination of the Tohoku Earthquake Impact on African Markets Onur BIYIK ${ }^{1}$
}

\begin{abstract}
This paper examines the Japanese International Bilateral Trade Flow (JIBTF) in relation to economic crises, such as the Tohoku earthquake, and focuses on changes in the Distribution Networks of JIBTF (DNoJ) concerning African countries and Japanese Major Trade Partners (JMTP). The Harmonized System 6-digit level ("total" and product level) panel data from 2001 to 2019 are analyzed by employing the (structural) gravity method. First, the results of the study find that the Tohoku earthquake, compared to the 2008 crisis, has a stronger negative impact on the JIBTF and affects the DNoJ among Japanese trade partners. Second, the results prove that Japanese trade intention with African markets decreases relatively. In other words, exporting to African countries has a negative interaction effect after 2015. On the contrary, this interaction effect on JMTP comparatively increases; thus, Japan relocates its export to other markets such as JMTP. The Tohoku earthquake shaped the JIBTF to be more integrated with the Asia-Pacific and the EU regions, instead of African markets.
\end{abstract}

Keywords: Japanese international trade, panel data, gravity model, Tohoku earthquake, African markets JEL Classification Codes: F10, F14, C23, C24

\footnotetext{
${ }^{1}$ PhD Program, Graduate School of Asia-Pacific Studies, Waseca University, 1-21-1 Nishiwaseda, Shinjuku, Tokyo, Japan, $165-$ 0051 (biyik-onur@fuji.waseda.jp)
} 


$\begin{array}{ll}\text { Abbreviations and Acronyms } \\ \text { DNoJ } & \text { Distribution Networks of Japanese International Bilateral Trade Flow } \\ \text { EPAs } & \text { Economic Partnership Agreements } \\ \text { FE } & \text { Fixed Effect } \\ \text { FTAs } & \text { Free Trade Agreements } \\ \text { GFC } & \text { Global Financial Crises } \\ \text { HS } & \text { Harmonized System } \\ \text { JIBTF } & \text { Japanese International Bilateral Trade Flow } \\ \text { JMTP } & \text { Japanese Major Trade Partners } \\ \text { OLS } & \text { Ordinary Least Squares } \\ \text { PPML } & \text { Poisson Pseudo-Maximum Likelihood } \\ \text { RE } & \text { Random Effect } \\ \text { TE } & \text { Tohoku Earthquake } \\ \text { ER } & \text { Export Recovery } \\ \text { EF } & \text { Export Fall }\end{array}$




\section{Introduction}

Japanese trade policy has evolved during the last two decades (2000-2020) (Mulgan \& Honma, 2015; Solis \& Urata, 2018). Japan has actively participated in bilateral and multilateral economic partnership agreements (EPAs) since 2002, increasing its participation to around $80 \%$ in terms of free trade agreements (FTAs) in total foreign trade (Biyik, 2021). The Comprehensive and Progressive Agreement for TransPacific Partnership and the Regional Comprehensive Economic Partnership are representable examples of Japanese trade policy regarding regional and global cooperation frameworks, strengthening the links between the Japanese economy and the world economy.

FTAs/EPAs boost the volume of bilateral and multilateral international trade flow (Arkolakis, Costinot, \& Rodríguez-Clare, 2012; Baier \& Bergstrand, 2007) and thereafter enhance sectoral interconnection between trading partners. This also applies to the spread of negative influence between countries through sectoral linkage. As examples of the Global Financial Crisis (GFC) (Acemoglu, Ozdaglar, \& Tahbaz-Salehi, 2015), the Tohoku Earthquake (TE) (Carvalho, Nirei, Saito, \& Tahbaz-Salehi, 2021), and other natural disasters (Barrot \& Sauvagnat, 2016; Raddatz, 2009) cause major adverse effects on macroeconomic variables worldwide. In other words, idiosyncratic shocks in multi-connected sectors (finance or energy) propagate negative macroeconomic impact among countries, transmitting negative shocks from the origin to all over the world (Acemoglu, Ozdaglar, \& Tahbaz-Salehi, 2017; Barrot \& Sauvagnat, 2016).

This reflects the fact that the geographical distribution of activities by Japanese firms/affiliates transfer any shock to (or from) other countries, thus, the Japanese economy faced various problems and bottlenecks due to the Lehman shock and the TE shock (Carvalho et al., 2021; Okiyama \& Tokunaga, 2017). These shocks had different impacts on the Japanese domestic market (Cavallo, Cavallo, \& Rigobon, 2014; Kajitani, Chang, \& Tatano, 2013) and then the Japanese International Bilateral Trade Flow (JIBTF) (Ando \& Kimura, 2012; Nanto, 2011). Therefore, this leads to changes in the Distribution Networks of JIBTF (DNoJ) among Japanese trading partners. In this context, this study aims to estimate the impact of (i) these shocks on JIBTF and aftermaths (ii) only TE shock on changes in Japanese trade distribution among its trading partners.

International trade studies regarding quantitative analyses examine by employing the (structural) gravity method (Head \& Mayer, 2014; Yotov, Piermartini, Monteiro, \& Larch, 2016), a common and most convenient tool for trade-related estimation (Larch, Wanner, Yotov, \& Zylkin, 2019; Yotov, Piermartini, Monteiro, \& Larch, 2017). Past literature focuses on the impact of the GFC and TE shocks on the Japanese market (Carvalho et al., 2021; Schnell \& Weinstein, 2012); however, the objective of this study is to estimate not only the impact of these shocks on JIBTF but also the impact of the earthquake on changes in the DNoJ 
regarding the African countries and Japanese Major Trade Partners (JMTP), in particular. To elucidate this purpose, this paper answers the following questions: Does the greatest damage to JIBTF come from either the 2008-2009 GFC or 2011 TE? To what extent does the earthquake affect DNoJ among Japanese trade partners? Is there a relocation of Japan's exports?

The paper first explains Japanese export and import trends by using country-product trade data (on yearly basis) at the most disaggregated level and the aggregated level. This is because the aim of this study empirically interprets the GFC/TE-related shocks. After that, this study focuses on the country characteristic variables and estimation techniques depending on the estimation method used. To examine two shocks' impacts on JIBTF, this study focuses on these shocks-related breaking points of bottom and peak in the aggregated dataset; thus, as an answer to the first question, this study presents that the TE shock has a stronger negative effect on JIBTF compared to the GFC shock.

Furthermore, to estimate the earthquake effects on DNoJ among Japanese trade partners, we track country year interaction in two datasets, as well as the probability of export fall and recovery in the disaggregated dataset. This means we examine whether or not Japan's export to JMTP and African countries increases/decreases regardless of regional preferences after the beginning of trade recovery from the TE. As an answer to the rest of the questions, it appears that Japanese trade intention with the African countries decreases relatively. Specifically, Japanese export to African countries has a negative interaction effect after the beginning of recovery from the earthquake. On the contrary, this interaction effect on JMTP comparatively increases; thus, Japan shifts its exports from African markets to other markets.

The reason is, is that being involved in the regional production networks among JMTP is more likely to sustain trade and achieve recovery from the TE shocks. Among African countries, those that have weak trade relationships in the production networks are not likely to recover from the TE shock. In short, the TE shock shaped the JIBTF to be more integrated with the Asia-Pacific and the EU regions, instead of African markets.

The rest of this paper is organized as follows: The second part introduces the background and literature to elucidate the working logic. The third section provides an explanation of this paper's datasets and portrays the data-related methodology and the empirical models. The fourth section discusses the empirical results of the two datasets. The last section concludes this study.

\section{Background and Literature}

Figure 1 shows Japanese export to its partners (lines as seen in different colors) as well as total export and import (bars as seen in red and blue). The figure presents the V-shape recovery of 2008-2009 GFC in 
terms of total export and import, seeing an initial peak in 2008 and a bottom in 2009. On the other hand, the TE shock displays a different pattern for export and import. For example, after the TE happened in 2011, while export immediately decreased, imports increased till 2012 and thereafter decreased. One of the reasons is that the Japanese limited energy resources caused a decrease in production ${ }^{2}$ and demanded more import products due to the TE shock.

In general, after the TE (followed by a tsunami and the failure of the Fukushima Dai-ichi Nuclear Power Plan) happened, people from Tohoku prefectures have emigrated and manufacturing industries in the disaster-stricken areas suffered (Higuchi, Inui, Hosoi, Takabe, \& Kawakami, 2012). In other words, on the one hand, the collapse of many buildings and infrastructures, on the other hand, many missing/dead people did result in massive damage to the Japanese economy/society, costing around $¥ 16.9$ trillion (Carvalho et al., 2021); thus, there are permanent changes in the machinery (parts and components) exports, decreasing (automobile) final goods' export consequences of a sharp decline of production in the manufacturing and mining sectors (Ando \& Kimura, 2012). This is because adverse supply shocks from small and medium enterprises (SMEs) in disaster areas affect exports through production chains (Carvalho et al., 2021).

\section{Figure 1: Japanese Bilateral Export Flows, Total Import, and Total Export}

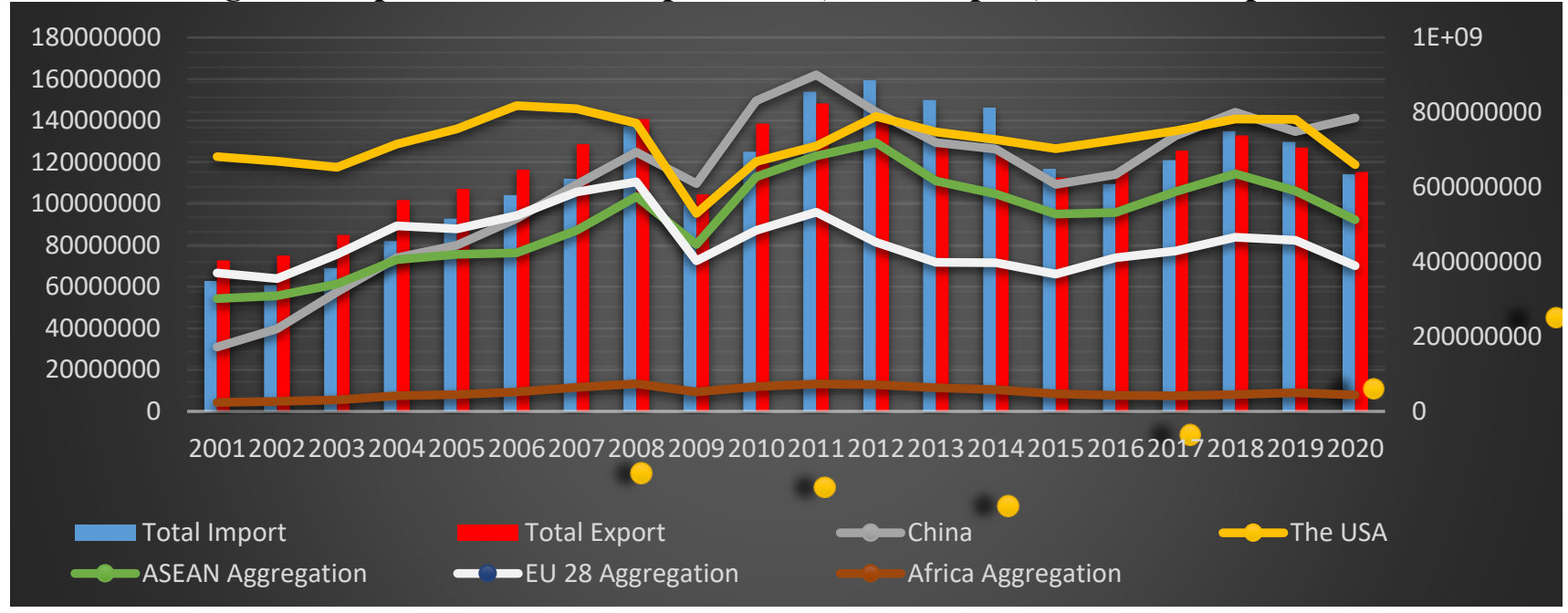

Note: Total import and export are based on the right axis. Japanese bilateral export flows are based on the left axis.

Source: Autor's preparation, the data available from the website of International Trade Center

To picture the TE shock with Figure 1, Japanese export and import (two bars, the right axis) decreased, respectively, till 2015 and 2016 as seen by (a different form of) V-shape ${ }^{3}$ line. After one year, the recovery of the trade from the TE shock saw in 2016 for export and in 2017 for import. Figure 1 also presents the number of exports-country pairs (five lines, the left axis). The largest Japanese exporting partners are China $(22 \%)$ and the USA (19\%), followed by ASEAN (15\%) and EU (12\%) members. Export to aggregated

\footnotetext{
${ }^{2}$ The four prefectures provide $4.5 \%$ of the Japanese output (Carvalho et al., 2021).

${ }^{3}$ As seen an initial peak in 2011/2012 and a bottom in 2015/2016 in respect to export/import (see Figure 1)
} 
African countries as a group (hereafter Africa) accounts for approximately $2 \%$ of total Japanese export over a 20 -year period.

In the literature, the impact of the natural disaster shock on the macro-economy is empirically investigated (Cavallo et al., 2014; Raddatz, 2009). As a firm-level study, Acemoglu et al., (2012), Acemoglu et al., (2017), and Baqaee and Farhi (2019) contribute propagation of microeconomic shock over firmslinkages within a country as well as across countries. Any natural disaster or financial shocks to the economy cause severe impacts on macroeconomic variables across the world (Acemoglu et al., 2015; Boehm, Flaaen, \& Pandalai-Nayar, 2019). Empirical evidence for these shocks with input-output linkages shows that macroeconomic volatility coming from sectoral idiosyncratic shocks is significantly large and diffuses/spreads (negative) cross-country transmission through multinational firms (Acemoglu, Akcigit, \& Kerr, 2016; Barrot \& Sauvagnat, 2016; Boehm et al., 2019). For example, the Tohoku earthquake not only harmed the Japanese economy (Carvalho et al., 2021) but also indirectly exposed the negative impact on the US economy through Japanese affiliates (Cavallo et al., 2014).

This study is similar to the approach of Noy (2009), Raddatz (2009), and Strobl (2012) articles, which comparatively estimate various disaster effects for developed and developing countries. As for the TEspecific studies, Schnell and Weinstein (2012) focus on comparative cases of the TE and Kobe earthquake and document that TE has more severe damages to Japanese industrial productions. This study analysis is closely related to the paper of Ando and Kimura (2012), which uses a similar monthly dataset from 2007 to 2011 and estimates comparisons of export changes under the GFC and TE shocks, especially machinery industries in Japan. That is, their focus, which is one of the references in this study, is Japanese machinery export volume changes across final and intermediate goods under GFC and TE shocks. They report that "GFC had huge and prolonged impacts, while the 2011 TE had much smaller and more temporary impacts, at least for Japan as a nation" (Ando \& Kimura, 2012). Their results, not all, are different than this paper. The limitation of their paper, which estimates the impact of two shocks on Japanese trade flow, is due to the monthly (seasonal and short-term) dataset.

This study uses annual datasets from 2001 to 2019 and covers all regions and all products. To update and contribute differently to this effect on the Japanese trade study, the main focus of this study estimates the earthquake effects on changes in the Japanese trade distribution network regarding African countries. Essentially, the originality of this study is to fill the gap where there are no studies on TE that take African countries into account, which is crucial for researchers/policymakers. In this sense, we constructed two datasets to examine the impact of GFC and TE shocks on (i) trade flows and the impact of TE shock on (ii) changes in trade network distribution among Japanese trading partners. 


\section{Methodology and Data}

This section first explains the outcome and explanatory variables related to using a specific method. Secondly, we introduce the general gravity model and thereafter build our empirical estimation model. Lastly, we report how to estimate our model with the datasets.

\subsection{Data Source}

This study uses two-panel datasets, Panel dataset A (aggregated level) and Panel dataset B (product level). Therefore, this subsection explains the datasets. In other words, we first portray outcome variables and then present explanatory variables associated with our method and country characteristics.

\subsubsection{The Outcome of Japanese Bilateral Trade Flow}

This paper's dependent variable as an outcome of Japanese bilateral trade data at the 6-digits level of the Harmonized System (HS) is extracted from International Trade Center-TradeMAp. The HS 6-digit level comprises approximately 5,300 articles/products descriptions and is aggregated to build as total-level (one sector/product) data. Accordingly, the total data represents a two-dimensional bilateral trade dataset (countries, aggregated sector, years), and HS 6-digit product-level data accounts for a three-dimensional bilateral trade dataset (countries, sectors, years). The two datasets at the total level and HS 6-digit productlevel contain, respectively, 3, 482 observations from 188 Japanese trading partners and 7,615,234 observations from 67 Japanese trading partners between 2001 and 2019 (see Table A1). In short, Panel dataset A and Panel dataset B account for roughly $99 \%$ of and $77 \%$ of the Japanese trade flows, respectively.

Moreover, we also check the impact of TE shock on the probability of trade fall and recovery concerning all products and manufacturing products as well as two regions in subsection 4.2.1., below. To formalize the features of Japanese exports in responding to the TE shock, we created binary variables such as export fall and export recovery. Technically, export fall presents 1 if no export of good $j$ to country $p$ in 2015 and 0 otherwise; in contrast, export recovery presents 1 if there is an export of good $J$ to country $p$ in 2016 and 0 otherwise as we explained the logic of breaking point of the Japanese export flows above. These combinations help this study to estimate whether Japanese exports (product-country pairs at the HS 6-digit level) ) existed in 2016, but not in 2015, addressing export recovery from the TE shock.

\subsubsection{Country Characteristics}

This study uses a number of broad country characteristic variables in the analysis that help us to explain cross-country variation. This study's independent variables include Gross Domestic Product (GDP), population, distance, each year dummy, import/export recovery year dummies, African dummy, and JMTP (as an expressed control group) dummy. Our primary use of these independent variables (i) satisfies the 
gravity method, (ii) helps to determine the effects of GFC and TE shocks, and (iii) estimates the impact of the TE shock on changes in the distribution of Japanese trade flows among its trading partners.

One would expect that big nations in economic size (a country with high GDP and population) have bigger foreign trade between each other. However, this integration becomes the opposite when the distance (trade cost) increases and then the trade amount between any two countries is diminished (Krugman \& Obstfeld, 2009). To estimate this integration, GDP (output) and population (expenditure/demand) data are taken from the World Development Indicators (World Bank) and Labor statistics over the years 2001-2019, respectively. We also obtained distance variables from CEPII GeoDist (Mayer \& Zignago, 2011) to quantify Japanese trade partners' geographic characteristics.

In the context of the impact of the economic crises, we question whether the greatest damage to Japanese trade flows comes from either GFC shock or TE shock. In other words, it is assumed that the 2011 TE shock has a stronger negative impact on JIBTF compared to the 2008 GFC shock. As for this purpose, we constructed each year dummy variable that accounts for a specific year by taking the value of one and zero otherwise (Year2009 = 1, otherwise “0”); thus, this study should be able to present each year's effect on JIBTF.

Furthermore, this study aims to empirically examine the effect of the TE shock on changes in DNoJ among Japanese trade partners. Our main hypothesis is that Japanese trade integration with the African countries decreases relatively. To comparatively explain this impact on African countries, we created African and control group (as presented by JMTP) dummies as regional preferences. Specifically, we constructed (i) African dummy (dmAfrica $\cong$ country belongs to African countries “1”, otherwise " 0 ”) and (i) control group dummy ( $d m J M T P \cong$ country belongs to Japanese major trade partners “1”, otherwise " 0 ”). This model comparatively examines two groups and then provides robust evidence to answer one of this paper's questions asked in this study.

Also, this study assumes that after the beginning of the trade recovery from the TE shock (2015 and 2016), Japan shifts its exports from African markets to other markets. To provide sufficient evidence for the interaction between recovery year and selected group countries, we first created (i) 2015 recovery dummy that explains a Japanese export recovery (dummy_ex2015 $\cong 2015 \geq 1$, otherwise " 0 ") and (ii) 2016 recovery dummy that presents a Japanese import recovery (dummy_im2016 $\cong 2016 \geq 1$, otherwise “0”) (see Figure1). Technically, interpreting interaction dummy explains 1 when African/JMTP dummy and recovery dummy equal to 1 (interaction of Africa equal 1 if dummy_ex2015 $=1 \&$ dmAfrica $=1$, otherwise " 0 "). All in all, two (selected) regional dummies associated with the recovery (year) dummies help us to comparatively present the regional integration effect. Also, the probability of exports to recover 
and fall with each country dummy indicates whether the country-product pair failed or survived after the TE.

\subsection{Methodology}

A gravity model is often regarded as the most popular and successful framework in international economics, highlighting many of the great future of theory-consistent empirical and political studies (Head \& Mayer, 2014; Yotov et al., 2016). Scholars such as Tinbergen (1962), Eaton-Kortum (2002), and Anderson and van Wincoop (2003) propose the theory of gravity equation to assess the main determinants of international trade. This study introduces the (traditional) gravity model ${ }^{4}$, as expressed in equation (1):

$$
Y_{o d}=\frac{\alpha_{0}\left(X_{o}^{\alpha_{1}}\right)\left(X_{d}^{\alpha_{2}}\right)}{D_{o d}^{\alpha_{3}}}
$$

Where: $Y_{o d}$ is the volumes of bilateral trade flow between the country of $o$ (origin) and the country of

$d$ (destination). $X_{o}^{\alpha_{1}} X_{d}^{\alpha_{2}}$ is to represent the national income of $o$ and $d$. Lastly, $D_{o d}^{\alpha_{3}}$ is a physical distance between the two trading partners.

A structure of the gravity model in panel data is written as a logarithmic form in equation (2):

$$
\boldsymbol{Y}_{o d, t}=\alpha_{0}+\alpha_{1} \boldsymbol{X}_{o, t}+\alpha_{2} \boldsymbol{X}_{d, t}-\alpha_{3} \boldsymbol{D}_{o d, t}+\boldsymbol{\varepsilon}_{o d, t}
$$

Where: $\boldsymbol{Y}_{o d, t}$ is $(\log )$ trade flow between partners regarding time period $(t=1,2, \ldots, T), \boldsymbol{X}$ is $(\log )$ explanatory variables, $\boldsymbol{D}$ is $(\log )$ destination (trade costs), $\alpha$ is structural parameters (elasticity), and $\boldsymbol{\varepsilon}$ is random component/error term

The recent development of the structural gravity model shows in equation (3):

$$
\boldsymbol{Y}_{o d, t}=\exp \left[\Pi_{o, t}+\chi_{d, t}+\alpha_{1} \boldsymbol{X}_{o, t}+\alpha_{2} \boldsymbol{X}_{d, t}-\alpha_{3} \boldsymbol{D}_{o d, t}\right]+\boldsymbol{\varepsilon}_{o d, t}
$$

The export-time, import-time, and pair fixed effects $\left(\Pi_{o, t}\right.$ and $\left.\chi_{d, t}\right)$ ensure the theoretical restrictions implied by structural gravity are satisfied (Larch et al., 2019).

\subsubsection{The Regression Speciation}

The general (panel) gravity model in this study accounts for output and expenditure, the measure of bilateral distance, and set of dummy variables for addressing the GFC and TE shocks, as well as the

${ }^{4}\left[\tau_{i j} /\left(\Pi_{i} P_{j}\right)\right]^{1-\sigma} Y_{i} E_{j} / Y:$ Where $Y_{i}, E_{j}$, and $Y$ are $i$ 's total income, $j$ 's total expenditure, and world total income respectively. $\left[\tau_{i j} /\left(\Pi_{i} P_{j}\right)\right]^{1-\sigma}$ is the trade cost term $(\sigma>1)$; thus, $\Pi_{i}$ and $P_{j}$ are $i$ 's outward multilateral resistance (weighted average of $i$ 's export trade costs) and $j$ 's inward multilateral resistance (weighted average of $j$ 's import trade costs) respectively, see Yotov et al., (2016) 
probability of export fall and recovery, as explained above. To answer this papers' questions, empirical models followed by Yotov et al., (2016) and Head and Mayer (2014) are determined.

\section{Equation of Economic Crises Effect on Trade Flow:}

$$
\begin{aligned}
& \text { Trade }_{J p n P, t}=\beta_{0}+\beta_{1} G D P_{J p n, t}+\beta_{2} G D P_{P, t}+\beta_{3} P_{J p n, t}+\beta_{4} P_{P, t}+\beta_{5} \text { Dist }_{J p n P} \\
& +\beta_{6} \sum_{t=2001}^{2019} \text { YearDummy }_{t}+\boldsymbol{\varepsilon}_{J p n P, t} \#(4) \\
& \operatorname{Trade}_{J p n P, t}=\exp \left[\beta_{0}+\beta_{1} G D P_{J p n, t}+\beta_{2} G D P_{P, t}+\beta_{3} P_{J p n, t}+\beta_{4} P_{P, t}+\beta_{5} \operatorname{Dist}_{J p n P}\right. \\
& \left.+\beta_{6} \sum_{t=2001}^{2019} \text { YearDummy }_{t}\right]+\boldsymbol{\varepsilon}_{J p n P, t} \#(5)
\end{aligned}
$$

\section{Equation of Trade Distributional Change:}

$$
\begin{aligned}
& \text { Trade }_{J p n P, t}=\beta_{0}+\beta_{1} G D P_{J p n, t}+\beta_{2} G D P_{P, t}+\beta_{3} P_{J p n, t}+\beta_{4} P_{P, t}+\beta_{5} D i s t_{J p n P} \\
& +\beta_{6} \text { RecoveryDummy }_{t}+\beta_{7} \text { RagionDummy }_{P, t} \\
& +\beta_{8} \text { RecoveryDummy }_{t} \# \text { RagionDummy }{ }_{P, t}+\boldsymbol{\varepsilon}_{J p n P, t} \#(6) \\
& \text { Trade }_{J p n P, t}=\exp \left[\beta_{0}+\beta_{1} G D P_{J p n, t}+\beta_{2} G D P_{P, t}+\beta_{3} P_{J p n, t}+\beta_{4} P_{P, t}\right. \\
& +\beta_{5} \text { Dist }_{\text {Jpn } P} \beta_{6} \text { RecoveryDummy }_{t}+\beta_{7} \text { RagionDummy }_{P, t} \\
& \left.+\beta_{8} \text { RecoveryDummy }_{t} \# \text { RagionDummy }{ }_{P, t}\right]+\boldsymbol{\varepsilon}_{J p n P, t} \#(7)
\end{aligned}
$$

\section{Probability of Export Fall and Recovery:}

$$
\begin{aligned}
& P\left(\text { EXchange }_{J p n P, t}=1\right) \\
& \quad=\Phi\left[\beta_{0}+\beta_{1} \text { Dist }_{J p n P}+\beta_{2} \text { Auto }_{J p n P, t}+\beta_{3} \sum_{p=1}^{P} \text { CountryDummy }_{P, t}\right]+\boldsymbol{\varepsilon}_{J p n P, t} \#(8)
\end{aligned}
$$

Where:

Trade $_{J p n P, t}$ represents Japanese total trade (Trade), Export (EX), and Import (IM) bilateral trade flow,

$E_{\text {Change }} e_{J p n P, t}$ is a binary variable relying on the exports fall and recovery regarding TE shock, $G D P_{J p n, t}$ and $G D P_{P, t}$ are the Japanese and its trade partner's GDP (constant $2010 \$$ ) as an output, $P O P_{J p n, t}$ and $P O P_{P, t}$ are the Japanese and its trade partner's population as an expenditure,

Distcap $_{J p n P}$ is the distance between Japanese capital to its trade partner capital, 
YearDummy $y_{t}$ is each year dummy from 2001 to 2019 ,

RecoveryDummy $y_{t}$ is the beginning of the trade recovery from the Tohoku earthquake (2015 for export and 2016 for import),

RagionDummy $_{P, t}$ is a group dummy of countries belonging to African countries/JMTP,

RecoveryDummy $_{t} \#$ RagionDummy ${ }_{P, t}$ is the interaction dummy between the recovery year and group of countries (Africa/JMTP),

CountryDummy $_{P, t}$ is each Japanese trade partner dummy,

Auto $_{P, t}$ accounts for the automobile sector as known sector 87 (Vehicles other than railway or tramway rolling stock, and parts and accessories thereof) regarding HS-2 level classification.

\subsubsection{Estimation Techniques}

This study is interested in presenting the impact of the GFC and TE shocks on (i) JIBTF and then the impact of the TE shock on (ii) changes in the DNoJ as well as the probability of export fall and recovery among Japanese trade partners. The volume of trade between two locations, both at the total and HS 6-digit level, is examined. This study separates the results of the two shocks based on a specific dataset. To address the impact of the two shocks on JIBTF, aggregated-level data is examined and represented in sub-section 4.1. After determining the highest impact from one of the shocks, two datasets (at the total and HS 6-digit level) are analyzed and interpreted changes in the DNoJ concerning African countries and JMTP (see Table A1), in particular.

Technically, since panel data is examined, this study uses the Housman test to identify whether the panel data estimation regarding two datasets implies a Random Effect (RE) or Fixed Effect (FE) (Wooldridge, 2016). As a result, while product-level estimation cannot reject the RE, the total-level estimation cannot prove if the RE is appropriate. Moreover, since this study uses differential weights data regarding the country level that cause/induce unequal variation (heteroscedasticity), thus, the cluster/robust is implied to adjust standard error for the fact that each of the units is individually weighted appropriately (Cameron, Gelbach, \& Miller, 2011; Egger \& Tarlea, 2015).

In addition, an aggregated gravity model with product-level data is another challenge for an estimation of this study. This is because bilateral trade contains more "0" (trade) values in the HS 6-digit product-level dataset. To address zero trade flow in disaggregated trade data as documented by Silva and Tenreyro (2006; 
2010), the Poisson Pseudo-Maximum Likelihood $\left(\mathrm{PPML}^{5}\right) /$ Poisson method is implemented with nested pair groups (Correia, Guimarães, \& Zylkin, 2019; Fally, 2015). As for the theory-consistent empirical work, multilateral resistance (export-time and import-time), which is partially FE by controlling country-year pair, is not appropriate to imply for pooled estimates of Ordinary Least Squares (OLS) and PPML models (Head \& Mayer, 2014; Yotov, 2016), as written in the modified equations (5) and (7); that is because the datasets account for only bilateral trade flow between Japan and its trade partners,

The last part of this paper analysis as a robustness check examines the probability of export trade fall and recovery (binary output/variable) by implying the probit/logit model relying on maximum likelihood estimation (MLE) (Long \& Freese, 2006) in subsection 4.2.1. We assume the standard cumulative normal/logistic distribution for $\Phi$ in the Probit model (logistic regression). Furthermore, this method is based on MLE and is not straightforward as OLS; thus, estimation command of the direction should be focused on such as whether they are statistically significant as well as positive/negative.

In general, every estimator has pros and cons in terms of its distributional characteristic and datasets. Therefore, this paper's results are presented through Between (long-run), Within (short-run), PPML, OLS, and Probit/Logit simulations because one method is not advisable (Gómez-Herrera, 2013). These (sensor data) estimation methods require dependent variables into size-adjusted (Silva \& Tenreyro, 2006). Therefore, all equations, dependent and independent variables, were divided by 100000 concerning trade values between 100 and 1000 as a maximum so that the log-likelihood function has values (Santos Silva, J M C \& Tenreyro, 2011; Silva \& Tenreyro, 2010).

\section{Result}

This section explains separately the impact of the GFC and TE shocks on JIBTF in the aggregated-level result and thereafter the impact of the TE shock on changes in the DNoJ among Japanese trading partners in two datasets result. The final analysis of export recovery and fall is presented in the product-level result.

\subsection{Aggregated-level Result}

The main purpose of this subsection is to first investigate which crises cause relatively stronger harm to Japanese total trade. Table 1 presents the impact of the GFC and the TE on JIBTF. Each row shows comparative cases of breaking points of trough/bottom and peak through the crises concerning between and within estimations (see Table $1 \&$ A2). To represent the specific main driver of trough and peak regarding export and import trade flow, it appeared that years dummies of 2009 and 2015/2016 (bottom of trade due

\footnotetext{
${ }^{5}$ Coding of the "ppmlhdfe" is a Stata package that implements the PPML with multi-way fixed effects. This function also allows scholars to code time variant variables (var1\#var2 or i.var1) (see Table 4; A2; A3; A4).
} 
to the GFC and TE shocks) have a negative impact on export and import; however, the year dummies of 2008 and 2011/2012 (peak of trade flow) have a crucial positive impact on export and import (see Table 1 and Figure 1)

Table 1: Comparing of GFC and TE shocks, Panel Estimates of Between and Within Effect

\begin{tabular}{|c|c|c|c|c|c|c|c|c|}
\hline VARIABLES & $\begin{array}{c}\text { Eq. } 4 \\
\text { RE } \\
\text { Trade }\end{array}$ & $\begin{array}{c}\text { Eq. } 4 \\
\text { FE } \\
\text { Trade }\end{array}$ & $\begin{array}{c}\text { Eq. } 4 \\
\text { RE } \\
\text { Trade }\end{array}$ & $\begin{array}{c}\text { Eq. } 4 \\
\text { FE } \\
\text { Trade }\end{array}$ & $\begin{array}{c}E q .4 \\
R E \\
E X \\
\end{array}$ & $\begin{array}{c}E q .4 \\
F E \\
E X \\
\end{array}$ & $\begin{array}{c}E q .4 \\
R E \\
I M \\
\end{array}$ & $\begin{array}{c}E q .4 \\
F E \\
I M \\
\end{array}$ \\
\hline$G D P p$ & $\begin{array}{c}0.00164 * * * \\
(0.000355)\end{array}$ & $\begin{array}{c}0.00179 * * * \\
(0.000384)\end{array}$ & $\begin{array}{c}0.00164 * * * \\
(0.000355)\end{array}$ & $\begin{array}{c}0.00179 * * * \\
(0.000384)\end{array}$ & $\begin{array}{c}0.000760^{* * * *} \\
(0.000111)\end{array}$ & $\begin{array}{c}0.000767 * * * \\
(0.000161)\end{array}$ & $\begin{array}{c}0.000895 * * * \\
(0.000237)\end{array}$ & $\begin{array}{c}0.00102 * * * \\
(0.000224)\end{array}$ \\
\hline$G D P j$ & $\begin{array}{c}2.94 \mathrm{e}-05 \\
(5.39 \mathrm{e}-05)\end{array}$ & $\begin{array}{c}0.000221^{* *} \\
(8.73 \mathrm{e}-05)\end{array}$ & $\begin{array}{c}0.000234 * * * \\
(6.61 \mathrm{e}-05)\end{array}$ & $\begin{array}{c}0.000254 * * * \\
(7.04 \mathrm{e}-05)\end{array}$ & $\begin{array}{c}8.83 \mathrm{e}-05 * * * \\
(3.41 \mathrm{e}-05)\end{array}$ & $\begin{array}{c}9.96 \mathrm{e}-05^{* * * *} \\
(3.43 \mathrm{e}-05)\end{array}$ & $\begin{array}{c}0.000144 * * * \\
(4.45 \mathrm{e}-05)\end{array}$ & $\begin{array}{c}0.000155^{* * * *} \\
(4.62 \mathrm{e}-05)\end{array}$ \\
\hline POPp & $\begin{array}{c}0.00252 \\
(0.00434)\end{array}$ & $\begin{array}{l}-0.00335 \\
(0.00481)\end{array}$ & $\begin{array}{c}0.00257 \\
(0.00433)\end{array}$ & $\begin{array}{l}-0.00317 \\
(0.00487)\end{array}$ & $\begin{array}{c}0.00147 \\
(0.00179)\end{array}$ & $\begin{array}{l}-1.35 \mathrm{e}-05 \\
(0.00209)\end{array}$ & $\begin{array}{c}0.00101 \\
(0.00255)\end{array}$ & $\begin{array}{l}-0.00316 \\
(0.00282)\end{array}$ \\
\hline$P O P j$ & $\begin{array}{c}0.00394 \\
(0.00256)\end{array}$ & $\begin{array}{c}0.157^{*} \\
(0.0825)\end{array}$ & $\begin{array}{c}0.143^{* * * *} \\
(0.0513)\end{array}$ & $\begin{array}{l}0.141 * * * \\
(0.0512)\end{array}$ & $\begin{array}{c}0.0730 * * * \\
(0.0261)\end{array}$ & $\begin{array}{c}0.0719 * * * \\
(0.0262)\end{array}$ & $\begin{array}{c}0.0699 * * \\
(0.0274)\end{array}$ & $\begin{array}{l}0.0687 * * \\
(0.0271)\end{array}$ \\
\hline distcap & $\begin{array}{c}-0.0782 * * * \\
(0.0278)\end{array}$ & & $\begin{array}{c}-0.0779 * * * \\
(0.0276)\end{array}$ & & $\begin{array}{c}-0.0354 * * \\
(0.0153)\end{array}$ & - & $\begin{array}{c}-0.0429 * * * \\
(0.0159)\end{array}$ & - \\
\hline Year2002 & $\begin{array}{l}-0.114 * \\
(0.0631)\end{array}$ & $\begin{array}{c}-0.565^{* *} * \\
(0.239)\end{array}$ & & & & & & \\
\hline Year2003 & $\begin{array}{c}0.110 \\
(0.207)\end{array}$ & $\begin{array}{c}-0.904 * * \\
(0.398)\end{array}$ & & & & & & \\
\hline Year2004 & $\begin{array}{l}0.614^{*} \\
(0.356)\end{array}$ & $\begin{array}{l}-0.689^{*} \\
(0.390)\end{array}$ & & & & & & \\
\hline Year2005 & $\begin{array}{c}0.918^{* * *} \\
(0.424)\end{array}$ & $\begin{array}{c}-0.572 \\
(0.466)\end{array}$ & & & & & & \\
\hline Year2006 & $\begin{array}{c}1.231 * * \\
(0.505)\end{array}$ & $\begin{array}{c}-0.532 \\
(0.540)\end{array}$ & & & & & & \\
\hline Year2007 & $\begin{array}{c}1.540 * * * \\
(0.583)\end{array}$ & $\begin{array}{l}-0.624 \\
(0.690)\end{array}$ & & & & & & \\
\hline Year2008 & $\begin{array}{c}2.493 * * * \\
(0.730)\end{array}$ & $\begin{array}{c}0.372 \\
(0.636)\end{array}$ & $\begin{array}{c}0.774 * * * \\
(0.175)\end{array}$ & $\begin{array}{c}0.765^{* * * *} \\
(0.175)\end{array}$ & $\begin{array}{l}0.315^{* * * *} \\
(0.0692)\end{array}$ & $\begin{array}{c}0.314 * * * \\
(0.0690)\end{array}$ & $\begin{array}{c}0.458 * * * \\
(0.139)\end{array}$ & $\begin{array}{c}0.451 * * * \\
(0.139)\end{array}$ \\
\hline Year2009 & $\begin{array}{c}0.526 \\
(0.411)\end{array}$ & $\begin{array}{l}-0.939 \\
(0.623)\end{array}$ & $\begin{array}{c}-0.532 * * \\
(0.271)\end{array}$ & $\begin{array}{l}-0.444 \\
(0.296)\end{array}$ & $\begin{array}{c}-0.408^{*} \\
(0.219)\end{array}$ & $\begin{array}{c}-0.367^{*} \\
(0.215)\end{array}$ & $\begin{array}{l}-0.127 \\
(0.148)\end{array}$ & $\begin{array}{r}-0.0769 \\
(0.135)\end{array}$ \\
\hline Year2010 & $\begin{array}{c}1.877 * * * \\
(0.705)\end{array}$ & $\begin{array}{c}-0.0550 \\
(0.589)\end{array}$ & & & & & & \\
\hline Year2011 & $\begin{array}{c}2.776^{* * * * * ⿰ ㇇ ⿰ 亅 ⿱ 丿 丶 丶 ~} \\
(0.893)\end{array}$ & $\begin{array}{l}1.229 * * \\
(0.494)\end{array}$ & $\begin{array}{c}1.561^{* * * *} \\
(0.436)\end{array}$ & $\begin{array}{c}1.612^{* * * *} \\
(0.450)\end{array}$ & $\begin{array}{c}0.557^{* * *} * \\
(0.232)\end{array}$ & $\begin{array}{c}0.581 * * \\
(0.231)\end{array}$ & $\begin{array}{c}1.002 * * * \\
(0.262)\end{array}$ & $\begin{array}{c}1.031 * * * \\
(0.268)\end{array}$ \\
\hline Year2012 & $\begin{array}{c}2.589 * * * \\
(0.806)\end{array}$ & $\begin{array}{c}1.206 \text { **** } \\
(0.366)\end{array}$ & $\begin{array}{c}1.483 * * * \\
(0.415)\end{array}$ & $\begin{array}{c}1.525^{* * * *} \\
(0.422)\end{array}$ & $\begin{array}{c}0.392^{* *} \\
(0.174)\end{array}$ & $\begin{array}{c}0.411 * * \\
(0.176)\end{array}$ & $\begin{array}{c}1.089 * * * \\
(0.287)\end{array}$ & $\begin{array}{c}1.114 * * * \\
(0.292)\end{array}$ \\
\hline Year2013 & $\begin{array}{c}1.849 * * * \\
(0.603)\end{array}$ & $\begin{array}{c}0.539 \\
(0.397)\end{array}$ & & & & & & \\
\hline Year2014 & $\begin{array}{c}1.454 * * * \\
(0.499)\end{array}$ & $\begin{array}{c}0.372 \\
(0.347)\end{array}$ & & & & & & \\
\hline Year2015 & $\begin{array}{c}0.100 \\
(0.182)\end{array}$ & $\begin{array}{c}-0.902 \text { ** } \\
(0.448)\end{array}$ & $\begin{array}{c}-0.758^{* * * *} * \\
(0.222)\end{array}$ & $\begin{array}{c}-0.738 * * * \\
(0.221)\end{array}$ & $\begin{array}{c}-0.407 \text { *** } * \\
(0.152)\end{array}$ & $\begin{array}{c}-0.399^{* * *} * \\
(0.152)\end{array}$ & $\begin{array}{c}-0.352 * * * \\
(0.102)\end{array}$ & $\begin{array}{c}-0.339 * * * \\
(0.103)\end{array}$ \\
\hline Year2016 & $\begin{array}{c}-0.296 * * \\
(0.138)\end{array}$ & $\begin{array}{c}-1.122 * * * \\
(0.397)\end{array}$ & $\begin{array}{c}-1.015^{* * *} \\
(0.269)\end{array}$ & $\begin{array}{c}-0.993^{* * *} * \\
(0.266)\end{array}$ & $\begin{array}{c}-0.394^{* * * *} \\
(0.141)\end{array}$ & $\begin{array}{c}-0.385^{* * * *} \\
(0.140)\end{array}$ & $\begin{array}{c}-0.621 \text { *** } \\
(0.164)\end{array}$ & $\begin{array}{c}-0.607 * * * \\
(0.163)\end{array}$ \\
\hline Year2017 & $\begin{array}{l}0.0871 \\
(0.164)\end{array}$ & $\begin{array}{c}-0.660^{* *} * \\
(0.288)\end{array}$ & & & & & & \\
\hline Year2018 & $\begin{array}{l}0.383^{*} \\
(0.200)\end{array}$ & - & & & & & & \\
\hline Year2019 & - & - & & & & & & \\
\hline Constant & & $\begin{array}{c}-211.7 * \\
(110.0)\end{array}$ & $\begin{array}{c}-187.9 * * * \\
(67.38)\end{array}$ & $\begin{array}{c}-192.5^{* * *} * \\
(69.12)\end{array}$ & $\begin{array}{c}-94.59 * * * \\
(34.62)\end{array}$ & $\begin{array}{c}-96.84 * * * \\
(35.48)\end{array}$ & $\begin{array}{c}-93.37 \text { **** } \\
(35.72)\end{array}$ & $\begin{array}{c}-95.65^{* * *} \\
(36.71)\end{array}$ \\
\hline Nested Cluster & Yes & Yes & Yes & Yes & Yes & Yes & Yes & Yes \\
\hline Observations & 3,482 & 3,482 & 3,482 & 3,482 & 3,482 & 3,482 & 3,482 & 3,482 \\
\hline$R$-squared & & 0.481 & & 0.479 & & 0.407 & & 0.452 \\
\hline Num. of Cou. & 188 & 188 & 188 & 188 & 188 & 188 & 188 & 188 \\
\hline
\end{tabular}

Note:(1)***,**,* represent significance at $1 \%, 5 \%$ and $10 \%$ level respectively; (2) standard errors in parentheses.

Source: Autor's calculation

Based on predicted results, although the GFC shock has a negative effect on JIBTF, the TE shock hasa comparatively stronger negative impact on JIBTF with statistically significant results. Therefore, the TE shock compared to the 2008 crisis has the strongest negative effect on total bilateral trade (see Table $1 \&$ 
Table A2). It can be said that the TE has become a severe economic crisis that hurt JIBTF more than the 2008 crisis.

It can be seen that export and import regarding the TE shock have (negative) significant correlations with Year2015 and Year2016, which show, respectively, the export and import (year) recovery from the TE shock (see Table $1 \&$ Table A2). That is because after the TE began, exports decreased while imports increased till 2012 as a consequence of recovering energy shortages (see Figure 1). Therefore, this (export and import recovery year) helps to track trade distributional changes by comparing African countries and JMTP in the production networks.

Table 2: Comparing of the Interaction Between Regions, Panel Estimates of Between and Within Effect in Aggregated-Level Data

\begin{tabular}{|c|c|c|c|c|c|c|c|c|}
\hline & (Africa) & (Africa) & (JMTP) & (JMTP) & (Africa) & (Africa) & (JMTP) & (JMTP) \\
\hline VARIABLES & $\begin{array}{c}E q .6 \\
R E \\
E X \\
\end{array}$ & $\begin{array}{c}E q .6 \\
R E \\
I M \\
\end{array}$ & $\begin{array}{c}E q .6 \\
R E \\
E X \\
\end{array}$ & $\begin{array}{c}E q .6 \\
R E \\
I M \\
\end{array}$ & $\begin{array}{c}E q .6 \\
F E \\
E X \\
\end{array}$ & $\begin{array}{c}E q .6 \\
F E \\
I M \\
\end{array}$ & $\begin{array}{c}E q .6 \\
F E \\
E X \\
\end{array}$ & $\begin{array}{c}E q .6 \\
F E \\
I M \\
\end{array}$ \\
\hline$G D P p$ & $\begin{array}{c}0.000765 * * * \\
(1.63 \mathrm{e}-05)\end{array}$ & $\begin{array}{c}0.000900 * * * \\
(2.02 \mathrm{e}-05)\end{array}$ & $\begin{array}{c}0.000768 * * * \\
(1.65 \mathrm{e}-05)\end{array}$ & $\begin{array}{c}0.000898 * * * \\
(2.04 \mathrm{e}-05)\end{array}$ & $\begin{array}{c}0.000773^{* * * *} \\
(1.97 \mathrm{e}-05)\end{array}$ & $\begin{array}{c}0.00103 * * * \\
(2.31 \mathrm{e}-05)\end{array}$ & $\begin{array}{c}0.000786^{* * * *} \\
(1.99 \mathrm{e}-05)\end{array}$ & $\begin{array}{c}0.00103 * * * \\
(2.35 \mathrm{e}-05)\end{array}$ \\
\hline$G D P j$ & $\begin{array}{c}0.000145 * * * \\
(2.65 \mathrm{e}-05)\end{array}$ & $\begin{array}{c}0.000213 * * * \\
(2.96 \mathrm{e}-05)\end{array}$ & $\begin{array}{c}0.000146 * * * \\
(2.64 \mathrm{e}-05)\end{array}$ & $\begin{array}{c}0.000213^{* * * *} \\
(2.96 \mathrm{e}-05)\end{array}$ & $\begin{array}{c}0.000156^{* * * *} \\
(2.66 \mathrm{e}-05)\end{array}$ & $\begin{array}{c}0.000223 * * * \\
(2.93 \mathrm{e}-05)\end{array}$ & $\begin{array}{c}0.000156^{* * * *} \\
(2.66 \mathrm{e}-05)\end{array}$ & $\begin{array}{c}0.000222 * * * \\
(2.93 \mathrm{e}-05)\end{array}$ \\
\hline POPp & $\begin{array}{c}0.00142 * * * \\
(0.000286)\end{array}$ & $\begin{array}{c}0.00100 * * * \\
(0.000381)\end{array}$ & $\begin{array}{c}0.00138 * * * \\
(0.000284)\end{array}$ & $\begin{array}{c}0.00106 * * * \\
(0.000379)\end{array}$ & $\begin{array}{c}-0.000144 \\
(0.000620)\end{array}$ & $\begin{array}{c}-0.00318 * * * \\
(0.000730)\end{array}$ & $\begin{array}{c}-0.000326 \\
(0.000621)\end{array}$ & $\begin{array}{c}-0.00315 * * * \\
(0.000732)\end{array}$ \\
\hline$P O P j$ & $\begin{array}{c}0.0549 * * * \\
(0.0142)\end{array}$ & $\begin{array}{c}0.0504 * * * \\
(0.0165)\end{array}$ & $\begin{array}{c}0.0550 * * * \\
(0.0142)\end{array}$ & $\begin{array}{c}0.0504 * * * \\
(0.0165)\end{array}$ & $\begin{array}{c}0.0547 * * * * \\
(0.0142)\end{array}$ & $\begin{array}{c}0.0497 * * * \\
(0.0163)\end{array}$ & $\begin{array}{c}0.0548^{* * * *} \\
(0.0141)\end{array}$ & $\begin{array}{c}0.0497 * * * \\
(0.0163)\end{array}$ \\
\hline distcap & $\begin{array}{c}-0.0402 * * * \\
(0.0125)\end{array}$ & $\begin{array}{c}-0.0505 * * * \\
(0.0172)\end{array}$ & $\begin{array}{c}-0.0274 * * \\
(0.0118)\end{array}$ & $\begin{array}{c}-0.0469 * * * \\
(0.0163)\end{array}$ & - & - & - & - \\
\hline 1.dummy_ex2015 & $\begin{array}{c}-0.805^{* * * *} \\
(0.211)\end{array}$ & & $\begin{array}{c}-0.437 * * \\
(0.209)\end{array}$ & & $\begin{array}{c}-0.802 * * * \\
(0.211)\end{array}$ & & $\begin{array}{c}-0.417 * * \\
(0.209)\end{array}$ & \\
\hline 1.dummy_im2016 & & $\begin{array}{c}-1.135 * * * \\
(0.248)\end{array}$ & & $\begin{array}{c}-0.974 * * * \\
(0.246)\end{array}$ & & $\begin{array}{c}-1.187 * * * \\
(0.244)\end{array}$ & & $\begin{array}{c}-0.897 * * * \\
(0.242)\end{array}$ \\
\hline 1.dmAfrica & $\begin{array}{c}0.712 \\
(0.986)\end{array}$ & $\begin{array}{c}1.341 \\
(1.364)\end{array}$ & & & - & - & & \\
\hline 1.dmJMTP & & & $\begin{array}{c}2.498 * * \\
(1.019)\end{array}$ & $\begin{array}{l}-1.173 \\
(1.408)\end{array}$ & & & - & - \\
\hline 1.dummy_ex2015\#1.dmAfrica & $\begin{array}{c}0.523 * * \\
(0.214)\end{array}$ & & & & $\begin{array}{c}0.549^{* *} \\
(0.215)\end{array}$ & & & \\
\hline 1.dummy_im2016\#1.dmAfrica & & $\begin{array}{l}0.498^{*} \\
(0.278)\end{array}$ & & & & $\begin{array}{c}0.696 * * \\
(0.274)\end{array}$ & & \\
\hline 1.dummy_ex2015\#1.dmJMTP & & & $\begin{array}{c}-1.008 * * * \\
(0.230)\end{array}$ & & & & $\begin{array}{c}-1.053 * * * \\
(0.232)\end{array}$ & \\
\hline 1.dummy_im2016\#1.dmJMTP & & & & $\begin{array}{c}-0.118 \\
(0.298)\end{array}$ & & & & $\begin{array}{l}-0.456 \\
(0.295)\end{array}$ \\
\hline Constant & $\begin{array}{c}-74.44 * * * \\
(18.24)\end{array}$ & $\begin{array}{c}-71.85^{* * *} \\
(21.45)\end{array}$ & $\begin{array}{c}-76.19 * * * \\
(18.21)\end{array}$ & $\begin{array}{c}-71.60 * * * \\
(21.49)\end{array}$ & $\begin{array}{c}-78.07 * * * \\
(18.18)\end{array}$ & $\begin{array}{c}-75.09 * * * \\
(21.03)\end{array}$ & $\begin{array}{c}-78.08^{* * * *} \\
(18.14)\end{array}$ & $\begin{array}{c}-75.13 * * * \\
(21.04)\end{array}$ \\
\hline Observations & 3,482 & 3,482 & 3,482 & 3,482 & 3,482 & 3,482 & 3,482 & 3,482 \\
\hline R-squared & & & & & 0.405 & 0.446 & 0.408 & 0.446 \\
\hline $\mathrm{F}$ & . & & . & . & 373.1 & 441.7 & 377.1 & 440.4 \\
\hline Number of Countries & 188 & 188 & 188 & 188 & 188 & 188 & 188 & 188 \\
\hline
\end{tabular}

Note:(1)***,**,* represent significance at 1\%,5\% and $10 \%$ level respectively; (2) standard errors in parentheses.

Source: Autor's calculation

The economic cycle concerning economic (crisis) shock causes different impacts such as changes in trade distribution. To portray the economic crisis-related trade distributional change among Japanese trade partners, this study's second focus is on the impact of the TE shock on African countries and JMTP in terms of export and import. Note that since recovery dummies result in a negative correlation, the interaction explanatory variables between recovery dummies and selected regions should be interpreted as opposite signs of the structural parameter (see Table 2). 
Predicted results show that African countries relatively receive more harm than JMTP regarding export. In other words, whereas exporting to JMTP increases (as seen in row 12, the blue color), exporting to Africa decreases (as seen in row 10, the red color) after the beginning of recovery from the TE shock (see Table 2; A3). Likewise, Japanese imports from African countries see a decrease through a significant result from the FE estimation (see Table 2); however, this study do not provide statistically significant results for Japanese imports from JMTP.

To bring this section to an end, the 2011 TE shock not only harms the JIBTF but also changes DNoJ among Japanese trade partners (see Table 1;2); thus, Japanese export to JMTP displays a significant positive correlation while this integration shows opposite signs between Japanese and African trade, decreasing Japanese export to African countries (see Table 2; A3).

\subsection{Product-level Result}

This subsection presents changes in the DNoJ between only two selected regions in the product-level dataset, which depends on only African countries and JMTP (the 28 EU countries aggregated as the EU (single) market). Therefore, the total number of country samples is 67-country which accounts for approximately $77 \%$ of Japanese trade.

Table 3: Comparing of the Interaction Between Regions, Panel Estimation of Between, Within Effect, and PPML in Product-Level Data

\begin{tabular}{|c|c|c|c|c|c|c|c|c|c|c|}
\hline VARIABLES & $\begin{array}{c}\text { (Africa) } \\
E q .6 \\
R E \\
E X \\
\end{array}$ & $\begin{array}{c}\text { (Africa) } \\
E q .6 \\
R E \\
I M \\
\end{array}$ & $\begin{array}{c}\text { (Africa) } \\
E q .6 \\
F E \\
E X \\
\end{array}$ & $\begin{array}{c}\text { (Africa) } \\
E q .6 \\
F E \\
I M \\
\end{array}$ & $\begin{array}{c}\text { (JMTP) } \\
E q .6 \\
R E \\
E X \\
\end{array}$ & $\begin{array}{c}\text { (JMTP) } \\
E q .6 \\
R E \\
I M \\
\end{array}$ & $\begin{array}{c}\text { (Africa) } \\
\text { Eq. } 7 \\
\text { Poisson }(\text { RE }) \\
\text { EX } \\
\end{array}$ & $\begin{array}{c}\text { (Africa) } \\
\text { Eq. } 7 \\
\text { Poisson }(R E) \\
I M \\
\end{array}$ & $\begin{array}{c}\text { (JMTP) } \\
\text { Eq. } 7 \\
\text { Poisson }(\text { RE) } \\
\text { EX } \\
\end{array}$ & $\begin{array}{c}\text { (JMTP) } \\
\text { Eq. } 7 \\
\text { Poisson (RE) } \\
\text { IM } \\
\end{array}$ \\
\hline GDPp & $\begin{array}{c}1.17 \mathrm{e}-09^{* * * *} \\
(0)\end{array}$ & $\begin{array}{c}1.18 \mathrm{e}-09^{* * * *} \\
(0)\end{array}$ & $\begin{array}{c}1.08 \mathrm{e}-09^{* * * *} \\
(0)\end{array}$ & $\begin{array}{c}1.54 \mathrm{e}-09^{* * * *} \\
(0)\end{array}$ & $\begin{array}{c}1.17 \mathrm{e}-09^{* * * *} \\
(0)\end{array}$ & $\begin{array}{c}1.18 \mathrm{e}-09^{* * * *} \\
(0)\end{array}$ & $\begin{array}{c}1.13 \mathrm{e}-08^{* * * *} \\
(2.41 \mathrm{e}-10)\end{array}$ & $\begin{array}{c}3.88 \mathrm{e}-09^{* * *} \\
(2.43 \mathrm{e}-10)\end{array}$ & $\begin{array}{c}1.13 \mathrm{e}-08 * * * \\
(2.41 \mathrm{e}-10)\end{array}$ & $\begin{array}{c}3.88 \mathrm{e}-09 * * * \\
(2.43 \mathrm{e}-10)\end{array}$ \\
\hline$G D P j$ & $\begin{array}{c}6.15 \mathrm{e}-10^{* * * *} \\
(0)\end{array}$ & $\begin{array}{c}6.65 \mathrm{e}-10^{* * * *} \\
(0)\end{array}$ & $\begin{array}{c}4.80 \mathrm{e}-10^{* * * *} \\
(0)\end{array}$ & $\begin{array}{c}4.66 \mathrm{e}-10^{* * * *} \\
(5.19 \mathrm{e}-11)\end{array}$ & $\begin{array}{c}6.15 \mathrm{e}-10^{* * * *} \\
(0)\end{array}$ & $\begin{array}{c}6.65 \mathrm{e}-10^{* * * *} \\
(0)\end{array}$ & $\begin{array}{c}5.05 \mathrm{e}-08^{* * * *} \\
(2.37 \mathrm{e}-09)\end{array}$ & $\begin{array}{c}9.33 \mathrm{e}-08 * * * \\
(2.41 \mathrm{e}-09)\end{array}$ & $\begin{array}{c}5.05 \mathrm{e}-08^{* * *} \\
(2.37 \mathrm{e}-09)\end{array}$ & $\begin{array}{c}9.33 \mathrm{e}-08^{* * * *} \\
(2.41 \mathrm{e}-09)\end{array}$ \\
\hline$P O P p$ & $\begin{array}{c}6.41 \mathrm{e}-06 * * * * \\
(4.75 \mathrm{e}-07)\end{array}$ & $\begin{array}{c}8.56 \mathrm{e}-06 * * * \\
(4.02 \mathrm{e}-07)\end{array}$ & $\begin{array}{c}2.07 \mathrm{e}-05^{* * * *} \\
(1.57 \mathrm{e}-06)\end{array}$ & $\begin{array}{c}1.50 \mathrm{e}-05^{* * * *} * \\
(1.90 \mathrm{e}-06)\end{array}$ & $\begin{array}{c}6.41 \mathrm{e}-06 * * * \\
(4.75 \mathrm{e}-07)\end{array}$ & $\begin{array}{c}8.56 \mathrm{e}-06 * * * \\
(4.02 \mathrm{e}-07)\end{array}$ & $\begin{array}{c}0.000101 * * * * \\
(3.64 \mathrm{e}-06)\end{array}$ & $\begin{array}{c}0.000188 * * * * \\
(4.20 \mathrm{e}-06)\end{array}$ & $\begin{array}{c}0.000101 * * * * \\
(3.64 \mathrm{e}-06)\end{array}$ & $\begin{array}{c}0.000188 * * * * \\
(4.20 \mathrm{e}-06)\end{array}$ \\
\hline$P O P j$ & $\begin{array}{c}0.000203^{* * * *} \\
(2.34 \mathrm{e}-05)\end{array}$ & $\begin{array}{c}0.000132 \text { **** } \\
(2.76 \mathrm{e}-05)\end{array}$ & $\begin{array}{c}0.000208 \text { *** } \\
(2.34 \mathrm{e}-05)\end{array}$ & $\begin{array}{c}0.000144 * * * \\
(2.76 \mathrm{e}-05)\end{array}$ & $\begin{array}{c}0.000203 * * * * \\
(2.34 \mathrm{e}-05)\end{array}$ & $\begin{array}{c}0.000132 * * * \\
(2.76 \mathrm{e}-05)\end{array}$ & $\begin{array}{l}0.0230 * * * \\
(0.00115)\end{array}$ & $\begin{array}{c}0.0179^{* * * *} \\
(0.00118)\end{array}$ & $\begin{array}{c}0.0230 * * * \\
(0.00115)\end{array}$ & $\begin{array}{l}0.0179^{* * * *} \\
(0.00118)\end{array}$ \\
\hline distcap & $\begin{array}{c}-0.0180 * * * \\
(0.00370)\end{array}$ & $\begin{array}{c}-0.0380 * * * \\
(0.00307)\end{array}$ & - & - & $\begin{array}{c}-0.0180 \text { **** } \\
(0.00370)\end{array}$ & $\begin{array}{c}-0.0380^{* * * * *} \\
(0.00307)\end{array}$ & $\begin{array}{c}-0.350^{* * * * *} \\
(0.0446)\end{array}$ & $\begin{array}{c}0.857^{* * * *} \\
(0.0483)\end{array}$ & $\begin{array}{c}-0.350 * * * \\
(0.0446)\end{array}$ & $\begin{array}{c}0.857 * * * \\
(0.0483)\end{array}$ \\
\hline 1.dummy_ex2015 & $\begin{array}{c}-0.00678^{* * * *} \\
(0.000460)\end{array}$ & & $\begin{array}{c}-0.00712^{* * *} * \\
(0.000463)\end{array}$ & & $\begin{array}{c}-0.00125^{* * * *} \\
(0.000346)\end{array}$ & & $\begin{array}{c}-0.196 * * * \\
(0.0156)\end{array}$ & & $\begin{array}{l}-0.129 * * \\
(0.0576)\end{array}$ & \\
\hline 1.dummy_im2016 & & $\begin{array}{c}-0.00305^{* * * *} \\
(0.000566)\end{array}$ & & $\begin{array}{c}-0.00582^{* * * *} \\
(0.000573)\end{array}$ & & $\begin{array}{c}-0.00234 * * * \\
(0.000412)\end{array}$ & & $\begin{array}{c}-0.179 * * * \\
(0.0154)\end{array}$ & & $\begin{array}{c}-0.634 * * * \\
(0.0616)\end{array}$ \\
\hline 1.dmAfrica & $\begin{array}{c}0.00157 \\
(0.00325)\end{array}$ & $\begin{array}{c}0.0267 * * * \\
(0.00272)\end{array}$ & - & - & & & $\begin{array}{c}-4.013 * * * \\
(0.0457)\end{array}$ & $\begin{array}{c}-4.601 * * * * \\
(0.0459)\end{array}$ & & \\
\hline 1.dmJMTP & & & & & $\begin{array}{l}-0.00157 \\
(0.00325)\end{array}$ & $\begin{array}{c}-0.0267 * * * * \\
(0.00272)\end{array}$ & & & $\begin{array}{c}4.013 * * * \\
(0.0457)\end{array}$ & $\begin{array}{c}4.601 * * * \\
(0.0459)\end{array}$ \\
\hline 1.dummy_ex2015\#1.dmAfrica & $\begin{array}{c}0.00553^{* * * * *} \\
(0.000401)\end{array}$ & & $\begin{array}{c}0.00578^{* * * *} \\
(0.000407)\end{array}$ & & & & $\begin{array}{c}0.0676 \\
(0.0566)\end{array}$ & & & \\
\hline 1.dummy_im2016\#1.dmAfrica & & $\begin{array}{c}0.000709 \\
(0.000518)\end{array}$ & & $\begin{array}{c}0.00410 \text { ***** } \\
(0.000529)\end{array}$ & & & & $\begin{array}{c}-0.455^{* * * *} \\
(0.0608)\end{array}$ & & \\
\hline 1.dummy_ex2015\#1.dmJMTP & & & & & $\begin{array}{c}-0.00553 * * * * \\
(0.000401)\end{array}$ & & & & $\begin{array}{l}-0.0676 \\
(0.0566)\end{array}$ & \\
\hline 1.dummy_im2016\#1.dmJMTP & & & & & & $\begin{array}{l}-0.000709 \\
(0.000518)\end{array}$ & & & & $\begin{array}{c}0.455^{* * * *} \\
(0.0608)\end{array}$ \\
\hline /lnalpha & & & & & & & $\begin{array}{l}2.063^{* * * *} \\
(0.00813)\end{array}$ & $\begin{array}{l}2.359 * * * \\
(0.00883)\end{array}$ & $\begin{array}{l}2.063^{* * * *} \\
(0.00813)\end{array}$ & $\begin{array}{l}2.359 * * * \\
(0.00883)\end{array}$ \\
\hline Constant & $\begin{array}{c}-0.275^{* * * *} \\
(0.0301)\end{array}$ & $\begin{array}{c}-0.187 * * * * \\
(0.0357)\end{array}$ & $\begin{array}{c}-0.300^{* * * *} \\
(0.0300)\end{array}$ & $\begin{array}{c}-0.218^{* * * *} \\
(0.0357)\end{array}$ & $\begin{array}{c}-0.273^{* * * *} \\
(0.0303)\end{array}$ & $\begin{array}{c}-0.161 \text { **** } \\
(0.0359)\end{array}$ & $\begin{array}{c}-35.95^{* * * *} \\
(1.491)\end{array}$ & $\begin{array}{c}-32.50 * * * \\
(1.546)\end{array}$ & $\begin{array}{c}-39.97 * * * \\
(1.490)\end{array}$ & $\begin{array}{c}-37.10^{* * *} \\
(1.546)\end{array}$ \\
\hline Observations & $7,615,234$ & $7,615,234$ & $7,615,234$ & $7,615,234$ & $7,615,234$ & $7,615,234$ & $7,615,234$ & $7,615,234$ & $7,615,234$ & $7,615,234$ \\
\hline Number of id & 412,856 & 412,856 & 412,856 & 412,856 & 412,856 & 412,856 & 412,856 & 412,856 & 412,856 & 412,856 \\
\hline Number of Countries & 67 & 67 & 67 & 67 & 67 & 67 & 67 & 67 & 67 & 67 \\
\hline
\end{tabular}


Table 3 shows two different estimation results regarding panel data analysis. While the interaction of the 1.dummy_ex2015\#1.dmAfrica (red color) variable relying on RE/FE estimation displays the significant result, PPML regarding Poisson method presents insignificant interaction for Japanese exporting to Africa countries (see Table 3). Explanatory variable of Japanese importing from African countries (red color in row 11) provides reverse results between RE/FE and Poisson, displaying coefficients with opposite signs (see Table 3).

Table 4: Mixed Estimates of HS 27 (Energy) and HS 87 (Automobile) in Product-Level Data

\begin{tabular}{|c|c|c|c|c|c|c|c|c|c|c|}
\hline & (Africa) & (Africa) & (Africa) & (Africa) & (JMTP) & (Africa) & (Africa) & (Africa) & (Africa) & (JMTP) \\
\hline VARIABLES & \begin{tabular}{|c|} 
Eq. 6 \\
OLS \\
EX 87 \\
\end{tabular} & $\begin{array}{c}\text { Eq. } 6 \\
R E \\
\text { EX } 87 \\
\end{array}$ & $\begin{array}{c}\text { Eq. } 7 \\
\text { Poisson (RE) } \\
\text { EX 87 } \\
\end{array}$ & $\begin{array}{c}E q .7 \\
P P M L(R E) \\
E X 87 \\
\end{array}$ & \begin{tabular}{|c|}
$E q .6$ \\
$R E$ \\
$E X 87$ \\
\end{tabular} & \begin{tabular}{|l} 
Eq. 6 \\
OLS \\
IM 27 \\
\end{tabular} & \begin{tabular}{|l}
$E q .6$ \\
$R E$ \\
$I M 27$ \\
\end{tabular} & $\begin{array}{c}\text { Eq. } 7 \\
\text { Poisson }(R E) \\
\text { IM 27 } \\
\end{array}$ & $\begin{array}{c}E q .7 \\
P P M L(R E) \\
I M 27 \\
\end{array}$ & \begin{tabular}{|l|}
$E q .6$ \\
$R E$ \\
$I M 27$ \\
\end{tabular} \\
\hline GDPp & $\begin{array}{c}2.40 \mathrm{e}-08 * * * \\
(7.24 \mathrm{e}-09)\end{array}$ & $\begin{array}{c}1.49 \mathrm{e}-08^{* * * *} \\
(8.04 \mathrm{e}-10)\end{array}$ & $\begin{array}{c}3.02 \mathrm{e}-09 * * \\
(1.18 \mathrm{e}-09)\end{array}$ & $\begin{array}{c}6.92 \mathrm{e}-10 \\
(4.89 \mathrm{e}-09)\end{array}$ & $\begin{array}{c}1.49 \mathrm{e}-08 * * * \\
(8.04 \mathrm{e}-10)\end{array}$ & $\begin{array}{c}-5.54 \mathrm{e}-09 \\
(4.10 \mathrm{e}-09)\end{array}$ & $\begin{array}{l}3.16 \mathrm{e}-09^{*} \\
(1.75 \mathrm{e}-09)\end{array}$ & $\begin{array}{c}6.61 \mathrm{e}-09 * * * \\
(1.89 \mathrm{e}-09)\end{array}$ & $\begin{array}{c}-2.78 \mathrm{e}-08^{* * * *} \\
(9.78 \mathrm{e}-09)\end{array}$ & $\begin{array}{l}3.16 \mathrm{e}-09^{*} \\
(1.75 \mathrm{e}-09)\end{array}$ \\
\hline$G D P j$ & $\begin{array}{c}4.93 \mathrm{e}-09^{* *} \\
(2.22 \mathrm{e}-09)\end{array}$ & $\begin{array}{c}7.37 \mathrm{e}-09 * * * \\
(1.88 \mathrm{e}-09)\end{array}$ & $\begin{array}{c}5.53 \mathrm{e}-08 * * * \\
(5.91 \mathrm{e}-09)\end{array}$ & $\begin{array}{c}6.99 \mathrm{e}-08 \text { *** } \\
(2.41 \mathrm{e}-08)\end{array}$ & $\begin{array}{c}7.37 \mathrm{e}-09^{* * * *} \\
(1.88 \mathrm{e}-09)\end{array}$ & $\begin{array}{c}3.54 \mathrm{e}-08 * * \\
(1.57 \mathrm{e}-08)\end{array}$ & $\begin{array}{c}3.36 \mathrm{e}-08 * * * \\
(4.44 \mathrm{e}-09)\end{array}$ & $\begin{array}{c}1.86 \mathrm{e}-07 * * * \\
(6.61 \mathrm{e}-09)\end{array}$ & $\begin{array}{c}2.11 \mathrm{e}-07 * * * \\
(2.53 \mathrm{e}-08)\end{array}$ & $\begin{array}{c}3.36 \mathrm{e}-08 * * * \\
(4.44 \mathrm{e}-09)\end{array}$ \\
\hline$P O P p$ & $\begin{array}{l}-5.56 \mathrm{e}-05 \\
(3.52 \mathrm{e}-05)\end{array}$ & $\begin{array}{l}-2.39 \mathrm{e}-06 \\
(2.56 \mathrm{e}-05)\end{array}$ & $\begin{array}{c}0.000365^{* * * *} \\
(6.05 \mathrm{e}-05)\end{array}$ & $\begin{array}{c}0.000236^{* * * *} \\
(6.29 \mathrm{e}-05)\end{array}$ & $\begin{array}{l}-2.39 \mathrm{e}-06 \\
(2.56 \mathrm{e}-05)\end{array}$ & $\begin{array}{c}4.21 \mathrm{e}-06 \\
(2.83 \mathrm{e}-05)\end{array}$ & $\begin{array}{l}-7.49 \mathrm{e}-05^{*} \\
(3.91 \mathrm{e}-05)\end{array}$ & $\begin{array}{c}-0.000242^{* * *} * \\
(5.62 \mathrm{e}-05)\end{array}$ & $\begin{array}{l}0.000207^{*} \\
(0.000119)\end{array}$ & $\begin{array}{l}-7.49 \mathrm{e}-05^{*} \\
(3.91 \mathrm{e}-05)\end{array}$ \\
\hline$P O P j$ & $\begin{array}{c}0.00241 * * \\
(0.00100)\end{array}$ & $\begin{array}{c}0.00225 * * \\
(0.00100)\end{array}$ & $\begin{array}{c}0.0158^{* * * *} \\
(0.00255)\end{array}$ & $\begin{array}{l}0.0152^{* *} \\
(0.00734)\end{array}$ & $\begin{array}{c}0.00225^{* *} \\
(0.00100)\end{array}$ & $\begin{array}{c}0.00355 \\
(0.00273)\end{array}$ & $\begin{array}{c}0.00341 \\
(0.00248)\end{array}$ & $\begin{array}{c}0.0284 * * * \\
(0.00324)\end{array}$ & $\begin{array}{c}0.0270 * * * \\
(0.00881)\end{array}$ & $\begin{array}{c}0.00341 \\
(0.00248)\end{array}$ \\
\hline distcap & $\begin{array}{l}0.207^{*} \\
(0.114)\end{array}$ & $\begin{array}{c}0.651^{* * *} * \\
(0.205)\end{array}$ & $\begin{array}{c}1.683^{* * *} \\
(0.216)\end{array}$ & $\begin{array}{c}4.529 * * * * \\
(1.241)\end{array}$ & $\begin{array}{c}0.651^{* * * *} \\
(0.205)\end{array}$ & $\begin{array}{c}0.765 \\
(0.615)\end{array}$ & $\begin{array}{l}0.298 \\
(0.299)\end{array}$ & $\begin{array}{l}-1.032 \\
(0.841)\end{array}$ & $\begin{array}{l}5.757^{* *} \\
(2.350)\end{array}$ & $\begin{array}{c}0.298 \\
(0.299)\end{array}$ \\
\hline 1.dummy_ex2015 & $\begin{array}{c}-0.124 \\
(0.0788)\end{array}$ & $\begin{array}{c}-0.0672 * * * * \\
(0.0198)\end{array}$ & $\begin{array}{c}-0.137 * * * * \\
(0.0351)\end{array}$ & $\begin{array}{c}-0.121 * * \\
(0.0503)\end{array}$ & $\begin{array}{c}-0.0140 \\
(0.0149)\end{array}$ & & & & & \\
\hline 1.dummy_im2016 & & & & & & $\begin{array}{l}-0.100 \\
(0.166)\end{array}$ & $\begin{array}{c}-0.157 * * * \\
(0.0511)\end{array}$ & $\begin{array}{c}-0.519 * * * \\
(0.0425)\end{array}$ & $\begin{array}{c}-0.509 * * * \\
(0.174)\end{array}$ & $\begin{array}{c}-0.128 * * * \\
(0.0370)\end{array}$ \\
\hline 1.dmAfrica & $\begin{array}{c}-0.306 * * * \\
(0.115)\end{array}$ & $\begin{array}{c}-0.775^{* * *} \\
(0.180)\end{array}$ & $\begin{array}{c}-4.132 * * * \\
(0.172)\end{array}$ & $\begin{array}{c}-6.366 * * * \\
(0.970)\end{array}$ & & $\begin{array}{l}-1.555^{*} \\
(0.813)\end{array}$ & $\begin{array}{c}-1.118^{* * * *} \\
(0.265)\end{array}$ & $\begin{array}{c}-3.293 * * * \\
(0.597)\end{array}$ & $\begin{array}{c}-8.216^{* * *} \\
(1.768)\end{array}$ & \\
\hline 1.dmJMTP & & & & & $\begin{array}{c}0.775^{* * * *} \\
(0.180)\end{array}$ & & & & & $\begin{array}{c}1.118 * * * \\
(0.265)\end{array}$ \\
\hline 1.dummy_ex2015\#1.dmAfrica & $\begin{array}{c}0.123 \\
(0.0839)\end{array}$ & $\begin{array}{c}0.0533 * * * \\
(0.0173)\end{array}$ & $\begin{array}{l}-0.156^{*} \\
(0.0902)\end{array}$ & $\begin{array}{l}-0.224 \\
(0.160)\end{array}$ & & & & & & \\
\hline 1.dummy_ex2015\#1.dmJMTP & & & & & $\begin{array}{c}-0.0533^{* * *} * \\
(0.0173)\end{array}$ & & & & & \\
\hline 1.dummy_im2016\#1.dmAfrica & & & & & & $\begin{array}{r}-0.0419 \\
(0.195)\end{array}$ & $\begin{array}{c}0.0289 \\
(0.0467)\end{array}$ & $\begin{array}{c}-1.220 * * * \\
(0.138)\end{array}$ & $\begin{array}{c}-1.383^{* * *} \\
(0.403)\end{array}$ & \\
\hline 1.dummy_im2016\#1.dmJMTP & & & & & & & & & & $\begin{array}{l}-0.0289 \\
(0.0467)\end{array}$ \\
\hline /lnalpha & & & $\begin{array}{l}2.018^{* * * *} \\
(0.0379)\end{array}$ & & & & & $\begin{array}{l}3.817 * * * \\
(0.0731)\end{array}$ & & \\
\hline Constant & $\begin{array}{c}-3.300 * * \\
(1.384)\end{array}$ & $\begin{array}{c}-3.322 * * \\
(1.296)\end{array}$ & $\begin{array}{l}-26.12 * * * * \\
(3.295)\end{array}$ & $\begin{array}{l}-27.60^{* * * *} \\
(10.28)\end{array}$ & $\begin{array}{c}-4.096 * * * \\
(1.313)\end{array}$ & $\begin{array}{l}-5.919 \\
(3.923)\end{array}$ & $\begin{array}{l}-5.477^{*} \\
(3.216)\end{array}$ & $\begin{array}{c}-45.89 * * * \\
(4.322)\end{array}$ & $\begin{array}{c}-49.60 * * * \\
(11.77)\end{array}$ & $\begin{array}{l}-6.595^{* * *} \\
(3.232)\end{array}$ \\
\hline Cluster Nested & Yes & No & No & Yes & No & Yes & No & No & Yes & No \\
\hline Observations & 114,796 & 114,796 & 114,796 & 114,796 & 114,796 & 55,449 & 55,449 & 55,449 & 55,449 & 55,449 \\
\hline $\mathrm{R}$-squared & 0.035 & & & & & 0.012 & & & & \\
\hline Number of id & 0 & 6,223 & 6,223 & 0 & 6,223 & 0 & 3,006 & 3,006 & 0 & 3,006 \\
\hline Number of Countries & 67 & 67 & 67 & 67 & 67 & 67 & 67 & 67 & 67 & 67 \\
\hline
\end{tabular}

Note:(1)***,**** represent significance at 1\%,5\% and 10\% level respectively; (2) standard errors in parentheses. OLS and PPML are Pooled estimations. RE and Poisson are panel results. IM 27: Mineral fuels, mineral oils, and products of their distillation; bituminous substances; mineral. IM 87: Vehicles other than railway or tramway rolling stock, and parts and accessories thereof Source: Autor's calculation

To improve this result and present the best fit estimation, pooled analysis (OLS and PPML) is also estimated (see Table A4 in the Appendix). As a result of the panel and pooled estimates, our estimations do not provide a similar result for Japanese trade distribution change between two regions (see Table 3; A4). For example, importing from Africa has a significant positive correlation through FE and OLS estimations (red color in row 11) while this integration variable turns out to be a negative correlation through Poisson and PPML estimations (see Table 3; A4). In summary, the fact that taking into account just value changes in each variable among countries-product pairs, exporting to African countries see a decrease (interaction) effect whereas exporting to JMTP comparatively increases after 2015 as seen in the 
first 6 columns, eq. 6 (see Table 3). this limitation led this study to use a different method focusing on each country-product pair distribution, as presented below in sub-section 4.2.1.

This subsection also investigates dominant sectors, which account for the Automobile sector for export and the energy sector for import, as questioning whether Japanese export dominant (EX 87) and import dominant (IM27) products have the same interaction effect after the trade recovery from the earthquake. Table 4 shows estimated results of these sectors' trade distribution change regarding African countries and JMTP. Panel estimation provides significant results that Japanese (EX 87) export to Africa decreases while Japanese (EX 87) export to JMTP increases. Also, energy-related (IM27) imports from African countries increase (see Table 4).

To summarize the findings in a few words, Japanese exports to Africa and JMTP have a significant correlation while this product-level estimation does not provide sufficient results for Japanese imports from both regions (see Table 3; A4). Therefore, the empirical results relying on RE/FE estimation convince us that Japan switched its export from African countries to JMTP after the beginning of the recovery from the earthquake in 2015 .

\subsubsection{Probability of Export Fall and Recovery}

This subsection further executes the previous analysis and comparatively presents the export changes among each selected country, instead of regional distribution. In other words, this paper uses another method with the binary outcome to track export changes (as of fall and recovery) based on selected countries such as China and Nigeria. Therefore, this method provides robust results and investigates country-based export fall and recovery concerning TE shock.

Primarily, we investigate the probability of the fall and recovery of export under TE shocks by implying the Logit/Probit estimation. The logic of the outcome variables is to examine whether there was no export in 2015 but export existed in 2016 for some countries, which represents export recovery from the TE shock. In general, Export Recovery (ER) presents there was a level of export in 2016, however, Export Fall (EF) accounts for the bottom of export in 2015 and 2016 and probability of no export at HS 6-digit level productcountry pair.

This study estimates separately EF and ER in different columns in terms of all products and manufacturing in 67 countries, as well as preferences of African (52) and JMTP (15) markets. Table 6 shows Probit/Logit (MLE) estimation of export fall and recovery and provides results of interesting findings. Note that although the Probit (MLE) estimation command of the direction of the effects (whether variables are statistically significant or positive/negative) is easy, the interpretation is not straightforward as OLS. 
However, the probability of each variable structural parameter regarding EF and ER provides a comparative important signal that we can measure as having a (relatively) high or low impact of TE shock.

First, the coefficient of China, the USA, and the EU are negative for the analysis of export fall and positive for the analysis of export recovery, with statistical significance at $1 \%$. This is because being involved in the regional production networks among JMTP is likely to sustain and recovery trade from the TE shock. Furthermore, as for coefficients of export fall and recovery, the EU and China comparatively have a probability of low recovery rate in manufacturing, as seen in (manufacturing) from the left side column $6^{\text {th }}$ (see Table 5).

Table 5: Panel Logit and Probit (MLE) Estimates of Japanese Export Change Under the TE

\begin{tabular}{|c|c|c|c|c|c|c|c|c|c|c|}
\hline & (ALL) & (ALL) & (ALL) & (ALL) & $\left(\right.$ Manfetr $\left.^{\mathbf{a}}\right)$ & $\left(\right.$ Manfetr $\left.^{\mathrm{a}}\right)$ & (Africa) & (Africa) & (JMTP) & (JMTP) \\
\hline VARIABLES & $\begin{array}{c}\text { Eq. } 8 \\
\text { Logit } \\
\text { ExFall } \\
\end{array}$ & $\begin{array}{c}\text { Eq. } 8 \\
\text { Logit } \\
\text { ExReco }\end{array}$ & \begin{tabular}{|l} 
Eq. 8 \\
Probit \\
ExFall \\
\end{tabular} & $\begin{array}{c}\text { Eq. } 8 \\
\text { Probit } \\
\text { ExReco }\end{array}$ & \begin{tabular}{|l} 
Eq. 8 \\
Probit \\
ExFall \\
\end{tabular} & $\begin{array}{c}\text { Eq. } 8 \\
\text { Probit } \\
\text { ExReco }\end{array}$ & \begin{tabular}{|c|} 
Eq. 8 \\
Probit \\
ExFall \\
\end{tabular} & $\begin{array}{c}\text { Eq. } 8 \\
\text { Probit } \\
\text { ExReco }\end{array}$ & $\begin{array}{c}\text { Eq. } 8 \\
\text { Probit } \\
\text { ExFall }\end{array}$ & $\begin{array}{c}\text { Eq. } 8 \\
\text { Probit } \\
\text { ExReco }\end{array}$ \\
\hline distcap & $\begin{array}{l}0.459^{* * * *} \\
(0.00558)\end{array}$ & $\begin{array}{c}-0.476 * * * \\
(0.00566)\end{array}$ & $\begin{array}{l}0.208 * * * \\
(0.00249)\end{array}$ & $\begin{array}{l}-0.214 * * * \\
(0.00251)\end{array}$ & $\begin{array}{l}0.329 * * * \\
(0.00658)\end{array}$ & $\begin{array}{c}-0.335^{* * *} * \\
(0.00663)\end{array}$ & $\begin{array}{c}0.0827 * * * \\
(0.0111)\end{array}$ & $\begin{array}{c}-0.109 * * * \\
(0.00521)\end{array}$ & $\begin{array}{c}0.163 * * * \\
(0.0133)\end{array}$ & $\begin{array}{c}-0.168 * * * \\
(0.0132)\end{array}$ \\
\hline Automobile & $\begin{array}{c}-0.271 * * * \\
(0.0159)\end{array}$ & $\begin{array}{c}0.263 * * * \\
(0.0160)\end{array}$ & $\begin{array}{l}-0.122 * * * \\
(0.00702)\end{array}$ & $\begin{array}{l}0.118 * * * \\
(0.00704)\end{array}$ & $\begin{array}{c}-0.0758 * * * \\
(0.00729)\end{array}$ & $\begin{array}{c}0.0726 * * * \\
(0.00732)\end{array}$ & $\begin{array}{c}-0.242^{* * * *} \\
(0.0170)\end{array}$ & $\begin{array}{l}0.109 * * * \\
(0.00764)\end{array}$ & $\begin{array}{c}-0.191 * * * \\
(0.0186)\end{array}$ & $\begin{array}{c}0.172 * * * \\
(0.0183)\end{array}$ \\
\hline China & $\begin{array}{c}-0.472 * * * \\
(0.0214)\end{array}$ & $\begin{array}{l}0.440 * * * \\
(0.0215)\end{array}$ & $\begin{array}{l}-0.195 * * * \\
(0.00875)\end{array}$ & $\begin{array}{l}0.181 * * * \\
(0.00876)\end{array}$ & $\begin{array}{c}-0.215 * * * \\
(0.0249)\end{array}$ & $\begin{array}{c}0.171 \text { **** } \\
(0.0243)\end{array}$ & & & $\begin{array}{c}-0.169 * * * * \\
(0.00930)\end{array}$ & $\begin{array}{l}0.170 * * * * \\
(0.00931)\end{array}$ \\
\hline The USA & $\begin{array}{c}-0.722 * * * \\
(0.0193)\end{array}$ & $\begin{array}{c}0.703 * * * \\
(0.0194)\end{array}$ & $\begin{array}{c}-0.315^{* * *} * \\
(0.00801)\end{array}$ & $\begin{array}{l}0.306 * * * \\
(0.00802)\end{array}$ & $\begin{array}{c}-0.458 * * * \\
(0.0231)\end{array}$ & $\begin{array}{c}0.458 * * * \\
(0.0233)\end{array}$ & & & $\begin{array}{c}-0.250 * * * \\
(0.0117)\end{array}$ & $\begin{array}{c}0.254 * * * \\
(0.0117)\end{array}$ \\
\hline The EU & $\begin{array}{c}-0.604 * * * * \\
(0.0191)\end{array}$ & $\begin{array}{c}0.568 * * * \\
(0.0191)\end{array}$ & $\begin{array}{c}-0.262 * * * \\
(0.00796)\end{array}$ & $\begin{array}{l}0.247 * * * \\
(0.00793)\end{array}$ & $\begin{array}{c}-0.402 * * * \\
(0.0234)\end{array}$ & $\begin{array}{c}0.375^{* * *} * \\
(0.0230)\end{array}$ & & & $\begin{array}{c}-0.206^{* * *} * \\
(0.00996)\end{array}$ & $\begin{array}{l}0.204^{* * * *} \\
(0.00993)\end{array}$ \\
\hline Liberia & $\begin{array}{c}-0.0871 \text { *** } \\
(0.0135)\end{array}$ & $\begin{array}{c}0.0607 * * * \\
(0.0135)\end{array}$ & $\begin{array}{c}-0.0399 * * * \\
(0.00624)\end{array}$ & $\begin{array}{c}0.0273 * * * \\
(0.00623)\end{array}$ & $\begin{array}{c}-0.0810 * * * \\
(0.0157)\end{array}$ & $\begin{array}{c}0.0624 * * * \\
(0.0156)\end{array}$ & $\begin{array}{c}-0.0321 * * \\
(0.0136)\end{array}$ & $\begin{array}{c}0.0120 * \\
(0.00626)\end{array}$ & & \\
\hline S.A. & $\begin{array}{c}-0.244 * * * \\
(0.0147)\end{array}$ & $\begin{array}{c}0.210 * * * \\
(0.0146)\end{array}$ & $\begin{array}{c}-0.112 * * * \\
(0.00661)\end{array}$ & $\begin{array}{c}0.0959 * * * \\
(0.00658)\end{array}$ & $\begin{array}{c}-0.264 * * * \\
(0.0183)\end{array}$ & $\begin{array}{c}0.236 * * * \\
(0.0180)\end{array}$ & $\begin{array}{c}-0.224 * * * \\
(0.0147)\end{array}$ & $\begin{array}{c}0.0902 * * * \\
(0.00658)\end{array}$ & & \\
\hline Egypt & $\begin{array}{l}0.00609 \\
(0.0143)\end{array}$ & $\begin{array}{c}-0.0388^{* * * *} \\
(0.0142)\end{array}$ & $\begin{array}{c}0.00180 \\
(0.00646)\end{array}$ & $\begin{array}{l}-0.0164 * * \\
(0.00644)\end{array}$ & $\begin{array}{c}-0.0948 * * * \\
(0.0177)\end{array}$ & $\begin{array}{c}0.0780 * * * \\
(0.0176)\end{array}$ & $\begin{array}{c}-0.122 * * * \\
(0.0146)\end{array}$ & $\begin{array}{c}0.0195^{* * *} \\
(0.00662)\end{array}$ & & \\
\hline Kenya & $\begin{array}{c}0.0115 \\
(0.0137)\end{array}$ & $\begin{array}{c}-0.0420 * * * \\
(0.0137)\end{array}$ & $\begin{array}{c}0.00444 \\
(0.00628)\end{array}$ & $\begin{array}{c}-0.0183 * * * \\
(0.00627)\end{array}$ & $\begin{array}{c}-0.0343 * * \\
(0.0162)\end{array}$ & $\begin{array}{c}0.0244 \\
(0.0162)\end{array}$ & $\begin{array}{c}-0.0527 * * * * \\
(0.0138)\end{array}$ & $\begin{array}{c}-0.000236 \\
(0.00631)\end{array}$ & & \\
\hline Nigeria & $\begin{array}{c}-0.0601 \text { **** } \\
(0.0137)\end{array}$ & $\begin{array}{c}0.0300 * * \\
(0.0136)\end{array}$ & $\begin{array}{c}-0.0280 * * * \\
(0.00628)\end{array}$ & $\begin{array}{l}0.0140 * * \\
(0.00626)\end{array}$ & $\begin{array}{c}-0.103 * * * \\
(0.0164)\end{array}$ & $\begin{array}{c}0.0757 * * * \\
(0.0162)\end{array}$ & $\begin{array}{c}-0.0594 * * * * \\
(0.0137)\end{array}$ & $\begin{array}{l}0.0139 * * \\
(0.00626)\end{array}$ & & \\
\hline Tanzania & $\begin{array}{c}0.0306 * * \\
(0.0135)\end{array}$ & $\begin{array}{c}-0.0500 * * * \\
(0.0135)\end{array}$ & $\begin{array}{l}0.0134 * * \\
(0.00621)\end{array}$ & $\begin{array}{c}-0.0222 * * * \\
(0.00621)\end{array}$ & $\begin{array}{c}-0.00231 \\
(0.0157)\end{array}$ & $\begin{array}{r}-0.00571 \\
(0.0158)\end{array}$ & $\begin{array}{c}-0.0179 \\
(0.0135)\end{array}$ & $\begin{array}{l}-0.00850 \\
(0.00624)\end{array}$ & & \\
\hline Morocco & $\begin{array}{c}0.0142 \\
(0.0136)\end{array}$ & $\begin{array}{c}-0.0356 * * * \\
(0.0136)\end{array}$ & $\begin{array}{c}0.00578 \\
(0.00625)\end{array}$ & $\begin{array}{l}-0.0156^{* * *} \\
(0.00625)\end{array}$ & $\begin{array}{c}-0.0275^{*} \\
(0.0161)\end{array}$ & $\begin{array}{c}0.0166 \\
(0.0160)\end{array}$ & $\begin{array}{c}-0.0399 * * * * \\
(0.0137)\end{array}$ & $\begin{array}{c}-0.000291 \\
(0.00629)\end{array}$ & & \\
\hline Constant & $\begin{array}{l}-3.502 * * * \\
(0.00664)\end{array}$ & $\begin{array}{l}3.542 * * * \\
(0.00674)\end{array}$ & $\begin{array}{c}-1.897 * * * \\
(0.00293)\end{array}$ & $\begin{array}{l}1.914 * * * \\
(0.00295)\end{array}$ & $\begin{array}{l}-2.073 * * * \\
(0.00787)\end{array}$ & $\begin{array}{l}2.089 * * * \\
(0.00794)\end{array}$ & $\begin{array}{c}-3.014 * * * \\
(0.0140)\end{array}$ & $\begin{array}{l}1.777 * * * \\
(0.00660)\end{array}$ & $\begin{array}{c}-1.913^{* * *} * \\
(0.00651)\end{array}$ & $\begin{array}{l}1.915 * * * \\
(0.00651)\end{array}$ \\
\hline $\begin{array}{l}\text { Observations } \\
\# \text { of id } \\
\# \text { of countries }\end{array}$ & $\begin{array}{c}7,615,234 \\
412,856 \\
67 \\
\end{array}$ & $\begin{array}{c}7,615,234 \\
412,856 \\
67 \\
\end{array}$ & $\begin{array}{c}7,615,234 \\
412,856 \\
67 \\
\end{array}$ & $\begin{array}{c}7,615,234 \\
412,856 \\
67 \\
\end{array}$ & $\begin{array}{c}1,281,543 \\
69,471 \\
67 \\
\end{array}$ & $\begin{array}{c}1,281,543 \\
69,471 \\
67 \\
\end{array}$ & $\begin{array}{c}5,968,694 \\
326,196 \\
52 \\
\end{array}$ & $\begin{array}{c}5,968,694 \\
326,196 \\
52 \\
\end{array}$ & $\begin{array}{c}1,646,540 \\
86,660 \\
15\end{array}$ & $\begin{array}{c}1,646,540 \\
86,660 \\
15\end{array}$ \\
\hline
\end{tabular}

Note:(1)******* represent significance at 1\%,5\% and $10 \%$ level respectively; (2) standard errors in parentheses. S.A. is the Sought Africa. ${ }^{\text {a. }}$ This covers sectors 84-88 in terms of HS 2-digit level classification. 84: Machinery, mechanical appliances, nuclear reactors, boilers; parts thereof; 85: Electrical machinery and equipment and parts thereof; sound recorders and reproducers, television; 86: Railway or tramway locomotives, rolling stock and parts thereof; railway or tramway track fixtures; 87: Vehicles other than railway or tramway rolling stock, and parts and accessories thereof; 88: Aircraft, spacecraft, and parts thereof.

Source: Autor's calculation

Second, among African countries, those that have strong trade relationships in the production networks are likely to achieve trade recovery from the TE shock. For example, the coefficients for Liberia, South Africa, and Nigeria are negative for the analysis of export fall and positive for the analysis of export recovery, with statistical significance; in contrast, the coefficients for Egypt, Kenya, Tanzania, and Morocco are positive for the analysis of export fall with statistical insignificant, and negative results 
for the analysis of export recovery with statistical significance (see Table 5). It can be said that Japan lost its trade intention with these four countries due to the weak(ened) relationships in the production networks.

In addition, the coefficient for the Automobile industry is negative for the analysis of export fall and positive for the analysis of export recovery, with statistical significance at $1 \%$. The fact that the probability of export fall rate is higher than the export recovery rate for the automobile sector, which indicates the lowest recovery rate in African markets as seen in (Africa) from right side column $3^{\text {rd }}$. To finalize this study, Japan finds alternative supply sources and relocate routes of the supply chains based on the dependency of each country's relationships in the production networks, unlike the JMTP where it is involved in network productions.

\section{Conclusion}

As a consequence of negative/positive shocks propagating from the origin to all over the world, the Japanese economy faced various problems and bottlenecks due to the 2008 GFC shock and the 2011 TE shock. In this sense, this paper comparatively examines the impact of the GFC and TE shocks on JIBTF and then investigates the impact of the TE shock on changes in the DNoJ among Japanese trading partners. HS 6-digit level ("total" and product level) time-series panel data from 2001 to 2019 is examined by employing the (structural) gravity method. This study conducts different estimations based on the distribution/characteristic of the output variables in the datasets

First, this study has found that the 2011 TE shock had a stronger negative effect on the Japanese total trade compared to the 2008 GFC shock. This means the TE has become a severe economic crisis that harmed JIBTF more than the 2008 crisis. Also, the result showed that the years of 2015 and 2016 (bottom of trade) had, respectively, (negative) significant/stronger correlations with export and import trade flow, which show the export and import recovery year after the (2011) TE.

Second, the nature and characteristics of the TE shock caused the change in DNoJ among Japanese trading partners. Regarding the impact of TE shock on trading partners, those involved in the regional production networks are more likely to sustain trade and recovery from the TE shock; thus, it appeared that Japanese trade intention with the African countries has decreased relatively. Specifically, Japanese export to African countries has had a negative interaction effect after the beginning of recovery from the earthquake in 2015. On the contrary, this interaction effect on JMTP has comparatively increased; thus, Japan has repositioned its exports from African markets to other markets after 2015.

Those that have strong trade relationships in the production networks such as JMTP are likely to maintain trade and help to recover trade from the TE shock. Otherwise, countries such as Egypt, Kenya, 
Tanzania, and Morocco that have weakened relationships in the production networks are likely to not recovery trade from the TE shock. To recapitulate the findings of estimated results, the TE shock shaped the JIBTF to be more integrated with the Asia-Pacific and the EU regions, instead of African markets.

As stated briefly, based on the interdependence of global production networks for each country, Japan can find alternative sources of supply and relocate the routes of its supply chains., unlike the JMTP where it is involved in network productions. This high dependency is another challenge for the Japanese economy if any negative shock comes from JMTP. Therefore, Japan considers investing in relationships in other markets such as Africa and managing its market focus from a global perspective, instead of regional preferences. This also contributes to the diversity of goods as well as markets, which not only boosts Japanese welfare/GDP but improves recovery dynamism from any negative shocks.

\section{Appendix}

\section{Table A1: Japanese Bilateral Trade Partners}

\begin{tabular}{|c|c|c|}
\hline \multicolumn{2}{|c|}{ African Countries } & $\begin{array}{l}\text { South Africa, Nigeria, Egypt, Morocco, Algeria, Mauritania, Ghana, Madagascar, Ethiopia, } \\
\text { Tunisia, Seychelles, Tanzania, the United Republic of, Kenya, Libya, State of Mozambique, } \\
\text { Burkina Faso, Zimbabwe, Sierra Leone, Gabon, Congo, the Democratic Republic of, Senegal, } \\
\text { Zambia, Somalia, Botswana, Uganda, Namibia, Malawi, Côte d'Ivoire, Sudan, Cameroon, } \\
\text { Mauritius, Togo, Mali, Saint Helena, Rwanda, Angola, Eswatini, Western Sahara, Guinea, } \\
\text { Congo, Burundi, Djibouti, Niger, Central African Republic, Lesotho, Comoros, Gambia, } \\
\text { Liberia, Chad, Sao Tome and Principe, Eritrea, Equatorial Guinea, Benin, French Southern } \\
\text { and Antarctic Territories, Guinea-Bissau, South Sudan, Cabo, Verde, Sudan }\end{array}$ \\
\hline \multirow{6}{*}{$\begin{array}{l}\text { Japanese } \\
\text { Major } \\
\text { Trade } \\
\text { Partners } \\
\text { (Control } \\
\text { Group) }\end{array}$} & The European Union (EU) & $\begin{array}{l}\text { Austria (AUT), Belgium (BEL), Cyprus (CYP), Czech Republic (CZE), Denmark (DNK), } \\
\text { Estonia (EST), Finland (FIN), France (FRA), Germany (DEU), Greece (GRC), Hungary } \\
\text { (HUN), Ireland (IRL), Italy (ITA), Latvia (LVA), Lithuania (LTU), Luxembourg (LUX), } \\
\text { Malta (MLT), Netherlands (NLD), Poland (POL), Portugal (PRT), Slovakia (SVK), Slovenia } \\
\text { (SVN), Spain (ESP), Sweden (SWE), Bulgaria (BGR), Croatia (HRV), Romania (ROU) }\end{array}$ \\
\hline & ASEAN & $\begin{array}{l}\text { Malaysia (MYS), Singapore (SGP), Brunei Darussalam (BRN), Vietnam (VNM), Cambodia } \\
(\text { KHM), Indonesia (IDN), Lao PDR (LAO), Philippines (PHL), Thailand (THA), Myanmar }\end{array}$ \\
\hline & China (CHN) & 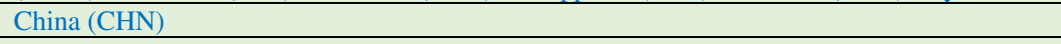 \\
\hline & United States (USA) & United States of America (USA) \\
\hline & Korea (KOR) & Republic of Korea (KOR) \\
\hline & Australia (AUS) & Australia (AUS) \\
\hline \multicolumn{2}{|c|}{ Rest of the World } & $\begin{array}{l}\text { Hong Kong, India, Mexico, Canada, Russian Federation, United Arab Emirates, Panama, } \\
\text { Saudi Arabia, Brazil, Turkey, New Zealand, Israel, Bangladesh, Kuwait, Oman, Marshall } \\
\text { Islands, Pakistan, Qatar, Chile, Colombia, Myanmar, Bahrain, Jordan, Argentina, Peru, Sri } \\
\text { Lanka, Iraq, Kazakhstan, Mongolia, Macao, Ecuador, Costa Rica, Bahamas, Yemen, Jamaica, } \\
\text { Dominican Republic, Papua New Guinea, Cyprus, Uzbekistan, United States Minor Outlying } \\
\text { Islands, Trinidad and Tobago, Georgia, Turkmenistan, Barbados, Lebanon, El Salvador, Iran, } \\
\text { Islamic Republic of, Tunisia, Paraguay, Honduras, Guyana, Azerbaijan, Suriname, } \\
\text { Nicaragua, Iceland, Nepal, New Caledonia, Fiji, Uruguay, Afghanistan, Macedonia, North, } \\
\text { Tuvalu, Belize, Serbia, French Polynesia, Maldives, Venezuela, Bolivarian Republic of, } \\
\text { Northern Mariana Islands, Micronesia, Federated States of, Haiti, Kyrgyzstan, Samoa, } \\
\text { Solomon Islands, Curaçao, Palau, Cuba, Cayman Islands, Vanuatu, Syrian Arab Republic, } \\
\text { Bermuda, Antigua and Barbuda, Armenia, Saint Lucia, Kiribati, Saint Kitts and Nevis, } \\
\text { Timor-Leste, Grenada, British Virgin Islands, Saint Vincent and the Grenadines, Somalia, } \\
\text { Moldova, Republic of, Tajikistan, Bhutan, Palestine, State of, Dominica, Montenegro, Nauru, } \\
\text { Cook Islands, Bosnia and Herzegovina, Turks and Caicos Islands, Albania, Anguilla, } \\
\text { Comoros, Montserrat, Andorra, Saint Helena, Greenland, Falkland Islands (Malvinas), } \\
\text { British Antarctic Territory, British Indian Ocean Territory, Faroe Islands, French Southern } \\
\text { and Antarctic Territories, Gibraltar, Korea, Democratic People's Republic of, Oceania Nes, } \\
\text { Niue, Africa not elsewhere specified, Pitcairn, America not elsewhere specified, St. Pierre } \\
\text { and Miquelon, Tokelau, Serbia and Montenegro, Area Nes. }\end{array}$ \\
\hline
\end{tabular}


Table A2: Comparing of GFC and TE shocks, Pooled Estimates of OLS and PPML with FE/RE

\begin{tabular}{|c|c|c|c|c|c|c|c|c|c|c|c|c|}
\hline $\begin{array}{l}\text { VARIAB } \\
\text { LES } \\
\end{array}$ & $\begin{array}{c}\text { Eq. } 5 \\
\text { PPML } \\
\text { Trade } \\
\end{array}$ & $\begin{array}{c}E q .5 \\
\text { PPML }(R E) \\
\text { Trade } \\
\end{array}$ & $\begin{array}{c}\text { Eq. } 5 \\
\text { PPML(FE) } \\
\text { Trade } \\
\end{array}$ & $\begin{array}{c}E q .5 \\
P P M L(R E) \\
E X \\
\end{array}$ & $\begin{array}{c}E q .5 \\
P P M L(F E) \\
E X \\
\end{array}$ & $\begin{array}{c}E q .5 \\
P P M L(R E) \\
l M \\
\end{array}$ & $\begin{array}{c}E q .5 \\
P P M L(F E) \\
l M \\
\end{array}$ & \begin{tabular}{|c|} 
Eq. 4 \\
OLS \\
Trade \\
\end{tabular} & $\begin{array}{c}\text { Eq. } 4 \\
\text { OLS } \\
\text { Trade } \\
\end{array}$ & \begin{tabular}{|c|} 
Eq. 4 \\
OLS \\
Trade \\
\end{tabular} & $\begin{array}{c}E q .4 \\
\text { OLS } \\
\text { EX } \\
\end{array}$ & $\begin{array}{c}E q .4 \\
\text { OLS } \\
\text { lM } \\
\end{array}$ \\
\hline$G D P p$ & $\begin{array}{c}2.69 \mathrm{e}-05^{* * *} * \\
(1.23 \mathrm{e}-06)\end{array}$ & $\begin{array}{c}2.69 \mathrm{e}-05^{* * * *} \\
(1.23 \mathrm{e}-06)\end{array}$ & $\begin{array}{l}-1.92 \mathrm{e}-06 \\
(2.88 \mathrm{e}-06)\end{array}$ & $\begin{array}{c}3.01 \mathrm{e}-05^{* * * *} \\
(1.29 \mathrm{e}-06)\end{array}$ & $\begin{array}{l}-3.42 \mathrm{e}-06 \\
(3.04 \mathrm{e}-06)\end{array}$ & $\begin{array}{c}2.35 \mathrm{e}-05^{* * * *} \\
(1.59 \mathrm{e}-06)\end{array}$ & $\begin{array}{c}8.67 \mathrm{e}-07 \\
(2.99 \mathrm{e}-06)\end{array}$ & $\begin{array}{c}0.00130^{* * * *} \\
(0.000162)\end{array}$ & $\begin{array}{c}0.00130^{* * * *} \\
(0.000161)\end{array}$ & $\begin{array}{c}0.00179^{* * * *} \\
(0.000394)\end{array}$ & $\begin{array}{c}0.000804 * * * \\
(5.23 \mathrm{e}-05)\end{array}$ & $\begin{array}{c}0.000497 * * * \\
(0.000129)\end{array}$ \\
\hline$G D P j$ & $\begin{array}{c}1.02 \mathrm{e}-05 \\
(3.52 \mathrm{e}-05)\end{array}$ & $\begin{array}{c}3.28 \mathrm{e}-05 \\
(3.70 \mathrm{e}-05)\end{array}$ & $\begin{array}{c}9.27 \mathrm{e}-05^{* * *} * \\
(1.70 \mathrm{e}-05)\end{array}$ & $\begin{array}{c}4.78 \mathrm{e}-06 \\
(4.19 \mathrm{e}-05)\end{array}$ & $\begin{array}{c}7.37 \mathrm{e}-05^{* * * * *} \\
(1.73 \mathrm{e}-05)\end{array}$ & $\begin{array}{l}6.01 \mathrm{e}-05^{*} \\
(3.36 \mathrm{e}-05)\end{array}$ & $\begin{array}{c}0.000113^{* * * *} \\
(1.61 \mathrm{e}-05)\end{array}$ & $\begin{array}{c}0.000186^{* * *} \\
(7.57 \mathrm{e}-05)\end{array}$ & $\begin{array}{c}0.000270^{* * * *} \\
(9.84 \mathrm{e}-05)\end{array}$ & $\begin{array}{c}0.000254^{* * * *} \\
(7.24 \mathrm{e}-05)\end{array}$ & $\begin{array}{c}7.60 \mathrm{e}-05^{*} \\
(4.32 \mathrm{e}-05)\end{array}$ & $\begin{array}{c}0.000194 * * * \\
(6.35 \mathrm{e}-05)\end{array}$ \\
\hline$P O P p$ & $\begin{array}{c}8.73 \mathrm{e}-05^{* * *} * \\
(2.89 \mathrm{e}-05)\end{array}$ & $\begin{array}{c}8.75 \mathrm{e}-05^{* * * *} \\
(2.89 \mathrm{e}-05)\end{array}$ & $\begin{array}{c}0.000503^{* *} \\
(0.000197)\end{array}$ & $\begin{array}{c}6.07 \mathrm{e}-05 \\
(3.78 \mathrm{e}-05)\end{array}$ & $\begin{array}{c}0.000702 * * * \\
(0.000239)\end{array}$ & $\begin{array}{c}0.000113^{* * *} \\
(3.05 \mathrm{e}-05)\end{array}$ & $\begin{array}{c}0.000215 \\
(0.000189)\end{array}$ & $\begin{array}{c}0.00538 \\
(0.00463)\end{array}$ & $\begin{array}{c}0.00538 \\
(0.00462)\end{array}$ & $\begin{array}{l}-0.00317 \\
(0.00500)\end{array}$ & $\begin{array}{c}0.00168 \\
(0.00172)\end{array}$ & $\begin{array}{c}0.00369 \\
(0.00291)\end{array}$ \\
\hline$P O P j$ & $\begin{array}{c}0.0379 * * * \\
(0.0128)\end{array}$ & $\begin{array}{c}0.0432 * * * \\
(0.0126)\end{array}$ & $\begin{array}{c}0.0250 * * * \\
(0.00651)\end{array}$ & $\begin{array}{c}0.0456 * * * \\
(0.0139)\end{array}$ & $\begin{array}{c}0.0247 * * * \\
(0.00752)\end{array}$ & $\begin{array}{c}0.0411 * * * \\
(0.0110)\end{array}$ & $\begin{array}{c}0.0259^{* * *} * \\
(0.00600)\end{array}$ & $\begin{array}{c}0.108 * * * * \\
(0.0360)\end{array}$ & $\begin{array}{c}0.135 * * * \\
(0.0458)\end{array}$ & $\begin{array}{c}0.141^{* * * *} \\
(0.0526)\end{array}$ & $\begin{array}{c}0.0726 * * * * \\
(0.0256)\end{array}$ & $\begin{array}{c}0.0623 * * * \\
(0.0229)\end{array}$ \\
\hline distcap & $\begin{array}{c}-0.0172^{* * * *} \\
(0.00383)\end{array}$ & $\begin{array}{c}-0.0172 * * * * \\
(0.00383)\end{array}$ & & $\begin{array}{c}-0.0198^{* * * *} * \\
(0.00543)\end{array}$ & & $\begin{array}{c}-0.0152^{* * * *} \\
(0.00351)\end{array}$ & & $\begin{array}{c}-0.0666 * * * \\
(0.0228)\end{array}$ & $\begin{array}{c}-0.0666^{* * * *} \\
(0.0228)\end{array}$ & $\begin{array}{l}-0.00656 \\
(0.0132)\end{array}$ & $\begin{array}{c}-0.0331 * * \\
(0.0148)\end{array}$ & $\begin{array}{c}-0.0335 * * * \\
(0.0111)\end{array}$ \\
\hline Year2002 & $\begin{array}{c}-0.149^{* * * *} \\
(0.0248)\end{array}$ & & & & & & & $\begin{array}{c}-0.471^{* * *} \\
(0.114)\end{array}$ & & & & \\
\hline Year2003 & $\begin{array}{c}-0.179^{* * * *} \\
(0.0338)\end{array}$ & & & & & & & $\begin{array}{c}-0.643 * * * \\
(0.184)\end{array}$ & & & & \\
\hline Year2004 & $\begin{array}{c}-0.0765^{* * *} * \\
(0.0283)\end{array}$ & & & & & & & $\begin{array}{c}-0.362^{* *} \\
(0.146)\end{array}$ & & & & \\
\hline Year2005 & $\begin{array}{l}-0.0189 \\
(0.0232)\end{array}$ & & & & & & & $\begin{array}{c}-0.188^{* * * *} \\
(0.0697)\end{array}$ & & & & \\
\hline Year2006 & $\begin{array}{l}0.00671 \\
(0.0169)\end{array}$ & & & & & & & $\begin{array}{l}-0.0426 \\
(0.0508)\end{array}$ & & & & \\
\hline Year2007 & - & & & & & & & - & & & & \\
\hline Year2008 & $\begin{array}{c}0.114 * \\
(0.0609)\end{array}$ & $\begin{array}{c}0.0858^{* * * *} \\
(0.0296)\end{array}$ & $\begin{array}{l}0.105 * * * \\
(0.0277)\end{array}$ & $\begin{array}{c}0.0660^{* * *} \\
(0.0267)\end{array}$ & $\begin{array}{c}0.0884 * * * * \\
(0.0253)\end{array}$ & $\begin{array}{c}0.105^{* * * * *} \\
(0.0393)\end{array}$ & $\begin{array}{c}0.119 \text { **** } \\
(0.0374)\end{array}$ & $\begin{array}{c}0.981 * * * \\
(0.252)\end{array}$ & $\begin{array}{c}0.794 * * * \\
(0.174)\end{array}$ & $\begin{array}{c}0.765^{*} * * \\
(0.180)\end{array}$ & $\begin{array}{c}0.316^{* * * * *} \\
(0.0696)\end{array}$ & $\begin{array}{c}0.478^{* * * * *} \\
(0.139)\end{array}$ \\
\hline Year2009 & $\begin{array}{l}-0.184 \\
(0.145)\end{array}$ & $\begin{array}{l}-0.140 \\
(0.111)\end{array}$ & $\begin{array}{c}0.0543 \\
(0.0653)\end{array}$ & $\begin{array}{l}-0.240^{*} \\
(0.131)\end{array}$ & $\begin{array}{c}-0.0279 \\
(0.0884)\end{array}$ & $\begin{array}{l}-0.0410 \\
(0.103)\end{array}$ & $\begin{array}{c}0.140^{* * * * *} \\
(0.0452)\end{array}$ & $\begin{array}{l}-0.507 \\
(0.384)\end{array}$ & $\begin{array}{l}-0.424 \\
(0.283)\end{array}$ & $\begin{array}{l}-0.444 \\
(0.304)\end{array}$ & $\begin{array}{l}-0.446^{*} \\
(0.258)\end{array}$ & $\begin{array}{c}0.0216 \\
(0.0843)\end{array}$ \\
\hline Year2010 & $\begin{array}{l}-0.00152 \\
(0.0927)\end{array}$ & & & & & & & $\begin{array}{c}0.488 \\
(0.358)\end{array}$ & & & & \\
\hline Year2011 & $\begin{array}{l}0.197 * \\
(0.111)\end{array}$ & $\begin{array}{l}0.202 * * \\
(0.0797)\end{array}$ & $\begin{array}{c}0.299 * * * \\
(0.0557)\end{array}$ & $\begin{array}{c}0.123 \\
(0.0883)\end{array}$ & $\begin{array}{c}0.222 * * * \\
(0.0720)\end{array}$ & $\begin{array}{c}0.279 * * * \\
(0.0809)\end{array}$ & $\begin{array}{c}0.373 * * * \\
(0.0439)\end{array}$ & $\begin{array}{c}1.676^{* * * *} * \\
(0.611)\end{array}$ & $\begin{array}{c}1.628 * * * \\
(0.519)\end{array}$ & $\begin{array}{c}1.612^{* * * *} * \\
(0.463)\end{array}$ & $\begin{array}{c}0.533 * * \\
(0.253)\end{array}$ & $\begin{array}{c}1.095 * * * \\
(0.313)\end{array}$ \\
\hline Year2012 & $\begin{array}{l}0.217 * * \\
(0.0897)\end{array}$ & $\begin{array}{c}0.213^{* * * *} \\
(0.0626)\end{array}$ & $\begin{array}{c}0.260 * * * \\
(0.0460)\end{array}$ & $\begin{array}{c}0.112 * \\
(0.0595)\end{array}$ & $\begin{array}{c}0.156^{* * * *} \\
(0.0477)\end{array}$ & $\begin{array}{c}0.306 * * * \\
(0.0669)\end{array}$ & $\begin{array}{c}0.357 * * * \\
(0.0445)\end{array}$ & $\begin{array}{c}1.586 \text { ***** } \\
(0.540)\end{array}$ & $\begin{array}{c}1.522 * * * \\
(0.458)\end{array}$ & $\begin{array}{c}1.525 * * * * \\
(0.434)\end{array}$ & $\begin{array}{c}0.373 * * \\
(0.177)\end{array}$ & $\begin{array}{c}1.149^{* * * *} \\
(0.322)\end{array}$ \\
\hline Year2013 & $\begin{array}{c}0.153 * * * \\
(0.0571)\end{array}$ & & & & & & & $\begin{array}{c}0.875 * * * \\
(0.330)\end{array}$ & & & & \\
\hline Year2014 & $\begin{array}{l}0.124^{* *} \\
(0.0582)\end{array}$ & & & & & & & $\begin{array}{c}0.645^{* *} * \\
(0.289)\end{array}$ & & & & \\
\hline Year2015 & $\begin{array}{c}-0.0757 * * * \\
(0.0268)\end{array}$ & $\begin{array}{c}-0.100 * * * \\
(0.0126)\end{array}$ & $\begin{array}{c}-0.104 * * * * \\
(0.0102)\end{array}$ & $\begin{array}{c}-0.115 * * * \\
(0.0245)\end{array}$ & $\begin{array}{c}-0.122 * * * * \\
(0.0210)\end{array}$ & $\begin{array}{c}-0.0876 * * * \\
(0.0229)\end{array}$ & $\begin{array}{c}-0.0860 * * * \\
(0.0266)\end{array}$ & $\begin{array}{c}-0.642 * * * \\
(0.184)\end{array}$ & $\begin{array}{c}-0.751^{* * * *} \\
(0.209)\end{array}$ & $\begin{array}{c}-0.738 * * * * \\
(0.227)\end{array}$ & $\begin{array}{c}-0.412 * * * \\
(0.151)\end{array}$ & $\begin{array}{c}-0.338 * * * \\
(0.0973)\end{array}$ \\
\hline Year2016 & $\begin{array}{c}-0.123 * * * * \\
(0.0233)\end{array}$ & $\begin{array}{c}-0.147 * * * * \\
(0.0189)\end{array}$ & $\begin{array}{c}-0.139^{* * * *} \\
(0.0226)\end{array}$ & $\begin{array}{c}-0.119 * * * \\
(0.0304)\end{array}$ & $\begin{array}{c}-0.113 * * * \\
(0.0254)\end{array}$ & $\begin{array}{c}-0.173^{* * * *} \\
(0.0301)\end{array}$ & $\begin{array}{c}-0.161 * * * \\
(0.0407)\end{array}$ & $\begin{array}{c}-0.894 * * * \\
(0.218)\end{array}$ & $\begin{array}{c}-0.989 * * * * \\
(0.251)\end{array}$ & $\begin{array}{c}-0.993^{* * * * *} * \\
(0.274)\end{array}$ & $\begin{array}{c}-0.396 * * * \\
(0.139)\end{array}$ & $\begin{array}{c}-0.593 * * * \\
(0.153)\end{array}$ \\
\hline Year2017 & $\begin{array}{c}-0.0441^{* * *} \\
(0.0165)\end{array}$ & & & & & & & $\begin{array}{c}-0.466^{* * * *} \\
(0.115)\end{array}$ & & & & \\
\hline Year2018 & $\begin{array}{c}0.0227 \\
(0.0146)\end{array}$ & & & & & & & $\begin{array}{l}0.0975 \\
(0.123)\end{array}$ & & & & \\
\hline Year2019 & - & & & & & & & - & & & & \\
\hline Constant & $\begin{array}{c}-46.13 * * * \\
(15.72)\end{array}$ & $\begin{array}{c}-54.29^{* * * *} \\
(16.09)\end{array}$ & $\begin{array}{c}-35.26 * * * \\
(8.973)\end{array}$ & $\begin{array}{c}-56.23 * * * \\
(18.24)\end{array}$ & $\begin{array}{c}-34.96 * * * \\
(10.56)\end{array}$ & $\begin{array}{c}-53.87^{* * * *} \\
(13.48)\end{array}$ & $\begin{array}{c}-37.29 * * * \\
(7.962)\end{array}$ & $\begin{array}{c}-141.4 * * * \\
(49.14)\end{array}$ & $\begin{array}{c}-181.0^{* * * *} \\
(63.23)\end{array}$ & $\begin{array}{c}-192.9^{* * * *} \\
(70.50)\end{array}$ & $\begin{array}{c}-93.80^{* * * *} \\
(34.35)\end{array}$ & $\begin{array}{c}-87.21^{* * * *} \\
(32.04)\end{array}$ \\
\hline$N C$ & Yes & Yes & Yes & Yes & Yes & Yes & Yes & Yes & Yes & Yes & Yes & Yes \\
\hline CDmmy & No & No & Yes & No & Yes & No & Yes & No & No & Yes & No & No \\
\hline$O b$. & 3,482 & 3,482 & 3,479 & 3,482 & 3,479 & 3,482 & 3,479 & 3,482 & 3,482 & 3,482 & 3,482 & 3,482 \\
\hline$R 2$ & & & & & & & & 0.757 & 0.757 & 0.966 & 0.808 & 0.628 \\
\hline Country & 188 & 188 & 188 & 188 & 188 & 188 & 188 & 188 & 188 & 188 & 188 & 188 \\
\hline
\end{tabular}

Note:(1)***,**** represent significance at $1 \%, 5 \%$ and $10 \%$ level respectively; (2) standard errors in parentheses. NC is nested clustered,

CDummy is country dummy,

Source: Autor's calculation 
Table A3: Comparing of the Interaction Between Regions, Pooled Estimates of OLS and PPML in Aggregated-Level Data

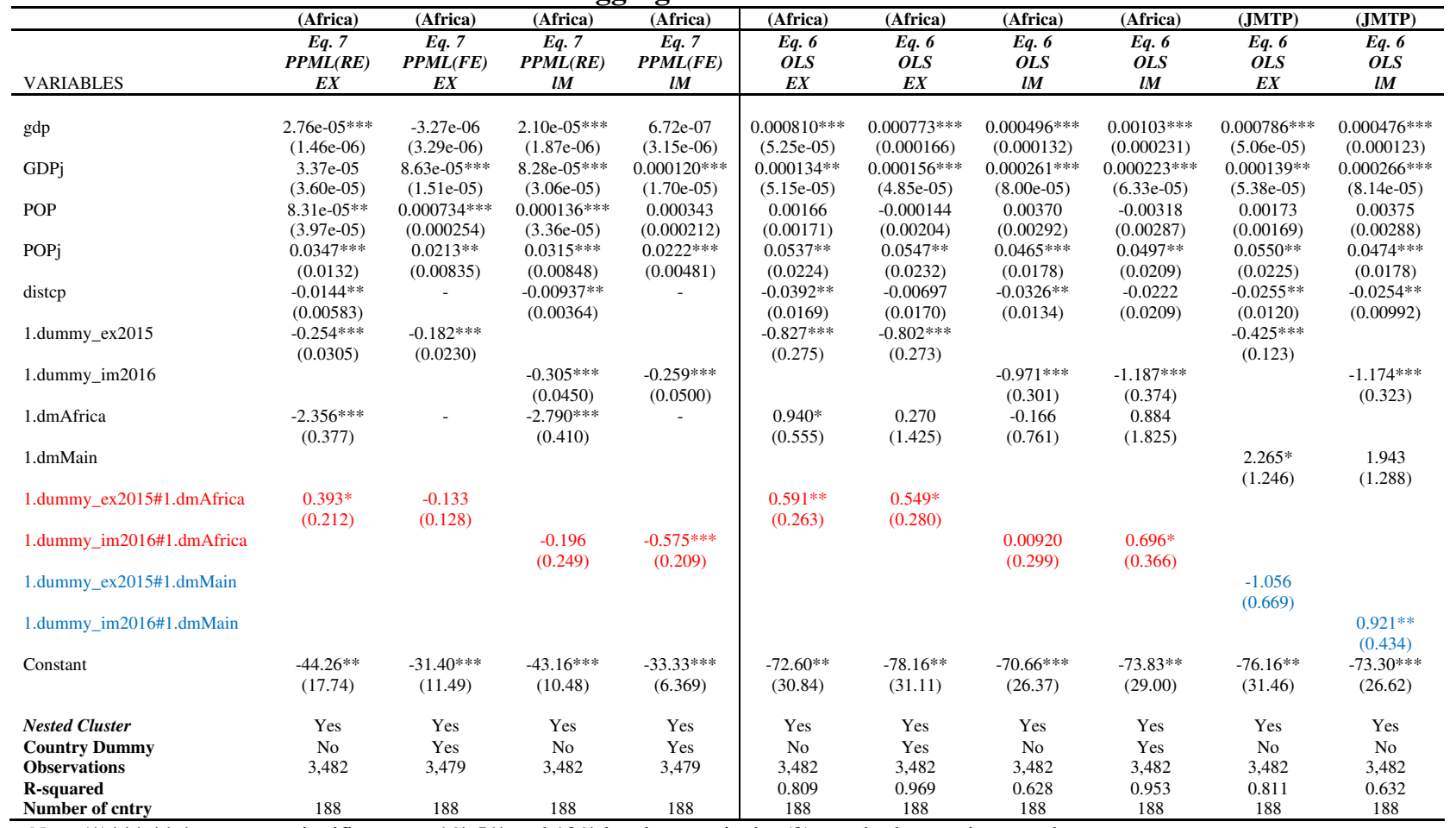

Note:(1)***,**,* represent significance at $1 \%, 5 \%$ and $10 \%$ level respectively; (2) standard errors in parentheses.

Source: Autor's calculation

Table A4: Comparing of the Interaction Between Regions, Pooled Estimates of OLS and PMML in Product-Level Data

\begin{tabular}{|c|c|c|c|c|c|c|c|c|}
\hline & (Africa) & (Africa) & (JMTP) & (JMTP) & (Africa) & (Africa) & (JMTP) & (JMTP) \\
\hline VARIABLES & $\begin{array}{c}E q .6 \\
O L S \\
E X \\
\end{array}$ & $\begin{array}{c}\text { Eq. } 6 \\
\text { OLS } \\
I M \\
\end{array}$ & $\begin{array}{c}E q .6 \\
O L S \\
E X \\
\end{array}$ & $\begin{array}{c}E q .6 \\
O L S \\
I M \\
\end{array}$ & $\begin{array}{c}E q .7 \\
P P M L(R E) \\
E X \\
\end{array}$ & $\begin{array}{c}E q .7 \\
P P M L(R E) \\
I M\end{array}$ & $\begin{array}{c}E q .7 \\
P P M L(R E) \\
E X\end{array}$ & $\begin{array}{c}E q .7 \\
P P M L(R E) \\
I M\end{array}$ \\
\hline GDPp & $\begin{array}{c}9.47 \mathrm{e}-10 \text { **** } \\
(2.14 \mathrm{e}-10)\end{array}$ & $\begin{array}{c}3.78 \mathrm{e}-10^{* * * *} \\
(7.25 \mathrm{e}-11)\end{array}$ & $\begin{array}{c}9.47 \mathrm{e}-10 * * * * \\
(2.14 \mathrm{e}-10)\end{array}$ & $\begin{array}{c}3.78 \mathrm{e}-10^{* * * *} \\
(7.25 \mathrm{e}-11)\end{array}$ & $\begin{array}{c}1.69 \mathrm{e}-08 * * * \\
(5.90 \mathrm{e}-09)\end{array}$ & $\begin{array}{c}3.71 \mathrm{e}-09 \\
(6.61 \mathrm{e}-09)\end{array}$ & $\begin{array}{c}1.69 \mathrm{e}-08 * * * * \\
(5.90 \mathrm{e}-09)\end{array}$ & $\begin{array}{c}3.71 \mathrm{e}-09 \\
(6.61 \mathrm{e}-09)\end{array}$ \\
\hline$G D P j$ & $\begin{array}{l}6.87 \mathrm{e}-10^{* * *} \\
(3.17 \mathrm{e}-10)\end{array}$ & $\begin{array}{l}8.81 \mathrm{e}-10^{* * *} \\
(4.21 \mathrm{e}-10)\end{array}$ & $\begin{array}{l}6.87 \mathrm{e}-10^{* * *} \\
(3.17 \mathrm{e}-10)\end{array}$ & $\begin{array}{c}8.81 \mathrm{e}-10^{* * *} \\
(4.21 \mathrm{e}-10)\end{array}$ & $\begin{array}{c}3.62 \mathrm{e}-08 \\
(2.68 \mathrm{e}-08)\end{array}$ & $\begin{array}{c}9.45 \mathrm{e}-08 * * * \\
(3.01 \mathrm{e}-08)\end{array}$ & $\begin{array}{c}3.62 \mathrm{e}-08 \\
(2.68 \mathrm{e}-08)\end{array}$ & $\begin{array}{c}9.45 \mathrm{e}-08 * * * * \\
(3.01 \mathrm{e}-08)\end{array}$ \\
\hline$P O P p$ & $\begin{array}{c}6.46 \mathrm{e}-06 * * * * \\
(1.49 \mathrm{e}-06)\end{array}$ & $\begin{array}{c}1.32 \mathrm{e}-05^{* * *} * \\
(7.89 \mathrm{e}-07)\end{array}$ & $\begin{array}{c}6.46 \mathrm{e}-06^{* * * *} \\
(1.49 \mathrm{e}-06)\end{array}$ & $\begin{array}{c}1.32 \mathrm{e}-05^{* * *} * \\
(7.89 \mathrm{e}-07)\end{array}$ & $\begin{array}{c}4.99 \mathrm{e}-05 \\
(6.52 \mathrm{e}-05)\end{array}$ & $\begin{array}{c}0.000181^{* *} \\
(7.30 \mathrm{e}-05)\end{array}$ & $\begin{array}{c}4.99 \mathrm{e}-05 \\
(6.52 \mathrm{e}-05)\end{array}$ & $\begin{array}{c}0.000181^{* *} \\
(7.30 \mathrm{e}-05)\end{array}$ \\
\hline$P O P j$ & $\begin{array}{l}0.000198^{*} \\
(0.000105)\end{array}$ & $\begin{array}{c}0.000125^{* *} \\
(5.73 \mathrm{e}-05)\end{array}$ & $\begin{array}{l}0.000198^{*} \\
(0.000105)\end{array}$ & $\begin{array}{c}0.000125^{* * *} \\
(5.73 \mathrm{e}-05)\end{array}$ & $\begin{array}{c}0.0251^{* * *} \\
(0.0123)\end{array}$ & $\begin{array}{l}0.0177 * * * \\
(0.00597)\end{array}$ & $\begin{array}{c}0.0251 * * \\
(0.0123)\end{array}$ & $\begin{array}{l}0.0177^{* * * *} \\
(0.00597)\end{array}$ \\
\hline distcap & $\begin{array}{c}-0.00793 \\
(0.0103)\end{array}$ & $\begin{array}{c}0.00215 \\
(0.00780)\end{array}$ & $\begin{array}{l}-0.00793 \\
(0.0103)\end{array}$ & $\begin{array}{c}0.00215 \\
(0.00780)\end{array}$ & $\begin{array}{l}-1.142 \\
(1.381)\end{array}$ & $\begin{array}{c}0.988 \\
(1.594)\end{array}$ & $\begin{array}{l}-1.142 \\
(1.381)\end{array}$ & $\begin{array}{c}0.988 \\
(1.594)\end{array}$ \\
\hline 1.dummy_ex2015 & $\begin{array}{c}-0.00532 * \\
(0.00286)\end{array}$ & & $\begin{array}{c}-0.00157^{* * * *} * \\
(0.000526)\end{array}$ & & $\begin{array}{c}-0.216^{* * *} \\
(0.0305)\end{array}$ & & $\begin{array}{l}-0.0408 \\
(0.147)\end{array}$ & \\
\hline 1.dummy_im2016 & & $\begin{array}{c}0.00215 \\
(0.00227)\end{array}$ & & $\begin{array}{c}-0.00356 * * \\
(0.00143)\end{array}$ & & $\begin{array}{c}-0.179 * * * \\
(0.0394)\end{array}$ & & $\begin{array}{c}-0.639 * * * * \\
(0.237)\end{array}$ \\
\hline 1.dmAfrica & $\begin{array}{l}-0.0114 * \\
(0.00663)\end{array}$ & $\begin{array}{l}-0.0159^{*} \\
(0.00918)\end{array}$ & & & $\begin{array}{c}-3.417 * * * \\
(0.985)\end{array}$ & $\begin{array}{c}-4.711 * * * \\
(1.244)\end{array}$ & & \\
\hline 1.dmJMTP & & & $\begin{array}{c}0.0114 * \\
(0.00663)\end{array}$ & $\begin{array}{c}0.0159^{*} \\
(0.00918)\end{array}$ & & & $\begin{array}{c}3.417 * * * \\
(0.985)\end{array}$ & $\begin{array}{c}4.711^{* * * *} \\
(1.244)\end{array}$ \\
\hline 1.dummy_ex2015\#1.dmAfrica & $\begin{array}{c}0.00375 \\
(0.00277)\end{array}$ & & & & $\begin{array}{c}0.175 \\
(0.164)\end{array}$ & & & \\
\hline 1.dummy_im2016\#1.dmAfrica & & $\begin{array}{c}-0.00572^{*} \\
(0.00301)\end{array}$ & & & & $\begin{array}{l}-0.460 * \\
(0.245)\end{array}$ & & \\
\hline 1.dummy_ex2015\#1.dmJMTP & & & $\begin{array}{l}-0.00375 \\
(0.00277)\end{array}$ & & & & $\begin{array}{l}-0.175 \\
(0.164)\end{array}$ & \\
\hline 1.dummy_im2016\#1.dmJMTP & & & & $\begin{array}{l}0.00572^{*} \\
(0.00301)\end{array}$ & & & & $\begin{array}{l}0.460^{*} \\
(0.245)\end{array}$ \\
\hline Constant & $\begin{array}{l}-0.272^{*} \\
(0.148)\end{array}$ & $\begin{array}{l}-0.199 * * \\
(0.0942)\end{array}$ & $\begin{array}{l}-0.283^{*} \\
(0.147)\end{array}$ & $\begin{array}{c}-0.215 * * \\
(0.0945)\end{array}$ & $\begin{array}{c}-37.39 * * \\
(16.31)\end{array}$ & $\begin{array}{c}-32.42 * * * \\
(7.548)\end{array}$ & $\begin{array}{c}-40.81^{* *} \\
(16.00)\end{array}$ & $\begin{array}{c}-37.13 * * * \\
(7.419)\end{array}$ \\
\hline $\begin{array}{l}\text { Cluster Nested } \\
\text { Observations }\end{array}$ & Yes & $\begin{array}{c}\text { Yes } \\
7,615,234\end{array}$ & Yes & Yes & $\begin{array}{c}\text { Yes } \\
7,615,234\end{array}$ & Yes & $\begin{array}{c}\text { Yes } \\
\end{array}$ & Yes \\
\hline Observations & $7,615,234$ & $7,615,234$ & $7,615,234$ & $7,615,234$ & $7,615,234$ & $7,615,234$ & $7,615,234$ & $7,615,234$ \\
\hline R-squared & 0.005 & 0.006 & 0.005 & 0.006 & 0.005 & 0.006 & 0.005 & 0.006 \\
\hline Number of Countries & 67 & 67 & 67 & 67 & 67 & 67 & 67 & 67 \\
\hline
\end{tabular}

Note:(1)***,**,* represent significance at $1 \%, 5 \%$ and $10 \%$ level respectively; (2) standard errors in parentheses.

Source: Autor's calculation 


\section{References}

Acemoglu, D., Akcigit, U., \& Kerr, W. (2016). Networks and the macroeconomy: An empirical exploration. Nber Macroeconomics Annual, 30(1), 273-335.

Acemoglu, D., Carvalho, V. M., Ozdaglar, A., \& Tahbaz-Salehi, A. (2012). The network origins of aggregate fluctuations. Econometrica, 80(5), 1977-2016.

Acemoglu, D., Ozdaglar, A., \& Tahbaz-Salehi, A. (2015). Systemic risk and stability in financial networks. American Economic Review, 105(2), 564-608.

Acemoglu, D., Ozdaglar, A., \& Tahbaz-Salehi, A. (2017). Microeconomic origins of macroeconomic tail risks. American Economic Review, 107(1), 54-108.

Anderson, J. E., \& Van Wincoop, E. (2003). Gravity with gravitas: A solution to the border puzzle. American Economic Review, 93(1), 170-192.

Ando, M., \& Kimura, F. (2012). How did the Japanese exports respond to two crises in the international production networks? the global financial crisis and the great east Japan earthquake. Asian Economic Journal, 26(3), 261-287.

Arkolakis, C., Costinot, A., \& Rodríguez-Clare, A. (2012). New trade models, same old gains? American Economic Review, 102(1), 94-130.

Baier, S. L., \& Bergstrand, J. H. (2007). Do free trade agreements actually increase members' international trade? Journal of International Economics, 71(1), 72-95.

Baqaee, D. R., \& Farhi, E. (2019). The macroeconomic impact of microeconomic shocks: Beyond Hulten's theorem. Econometrica, 87(4), 1155-1203.

Barrot, J., \& Sauvagnat, J. (2016). Input specificity and the propagation of idiosyncratic shocks in production networks. The Quarterly Journal of Economics, 131(3), 1543-1592.

Biyik, O. (2021). Japan-AfCFTA integration through economic impacts of alternative EPA scenariosexamination of the GTAP 10A MRIO database. doi:10.21203/rs.3.rs-762313/v1

Boehm, C. E., Flaaen, A., \& Pandalai-Nayar, N. (2019). Input linkages and the transmission of shocks: Firm-level evidence from the 2011 tōhoku earthquake. Review of Economics and Statistics, 101(1), 60-75.

Cameron, A. C., Gelbach, J. B., \& Miller, D. L. (2011). Robust inference with multiway clustering. Journal of Business \& Economic Statistics, 29(2), 238-249.

Carvalho, V. M., Nirei, M., Saito, Y. U., \& Tahbaz-Salehi, A. (2021). Supply chain disruptions: Evidence from the great east japan earthquake. The Quarterly Journal of Economics, 136(2), 1255-1321.

Cavallo, A., Cavallo, E., \& Rigobon, R. (2014). Prices and supply disruptions during natural disasters. Review of Income and Wealth, 60, S449-S471.

Correia, S., Guimarães, P., \& Zylkin, T. (2019). Ppmlhdfe: Fast poisson estimation with high-dimensional fixed effects, arXiv. org.

Eaton, J., \& Kortum, S. (2002). Technology, geography, and trade. Econometrica, 70(5), 1741-1779.

Egger, P. H., \& Tarlea, F. (2015). Multi-way clustering estimation of standard errors in gravity models. Economics Letters, 134, 144-147.

Fally, T. (2015). Structural gravity and fixed effects. Journal of International Economics, 97(1), 76-85.

Gómez-Herrera, E. (2013). Comparing alternative methods to estimate gravity models of bilateral trade. Empirical Economics, 44(3), 1087-1111.

Head, K., \& Mayer, T. (2014). Gravity equations: Workhorse, toolkit, and cookbook. Handbook of international economics (pp. 131-195) Elsevier.

Higuchi, Y., Inui, T., Hosoi, T., Takabe, I., \& Kawakami, A. (2012). The impact of the great east japan earthquake on the labor market - need to resolve the employment mismatch in the disaster-stricken areas. Japan Labor Review, 9(4), 4-21.

Kajitani, Y., Chang, S. E., \& Tatano, H. (2013). Economic impacts of the 2011 Tohoku-Oki earthquake and tsunami. Earthquake Spectra, 29(1_suppl), 457-478.

Krugman, P. R., \& Obstfeld, M. (2009). International economics: Theory and policy Pearson Education. 
Larch, M., Wanner, J., Yotov, Y. V., \& Zylkin, T. (2019). Currency unions and trade: A PPML reassessment with high-dimensional fixed effects. Oxford Bulletin of Economics and Statistics, 81(3), 487-510.

Long, J. S., \& Freese, J. (2006). Regression models for categorical dependent variables using stata Stata press.

Mayer, T., \& Zignago, S. (2011). Notes on CEPII's distances measures: The GeoDist database.

Mulgan, A. G., \& Honma, M. (2015). The political economy of Japanese trade policy Springer.

Nanto, D. K. (2011). Japan; s 2011 earthquake and tsunami: Economic effects and implications for the united states DIANE Publishing.

Okiyama, M., \& Tokunaga, S. (2017). Economic analysis of fiscal measures for reconstructing the Tohoku region after the great east japan earthquake: Using a dynamic two-regional CGE model. Spatial economic modeling of megathrust earthquake in japan (pp. 123-161) Springer.

Raddatz, C. E. (2009). The wrath of God: Macroeconomic costs of natural disasters. World Bank Policy Research Working Paper, (5039)

Santos Silva, J M C, \& Tenreyro, S. (2011). Further simulation evidence on the performance of the Poisson pseudo-maximum likelihood estimator. Economics Letters, 112(2), 220-222. doi:10.1016/j.econlet.2011.05.008

Schnell, M. K., \& Weinstein, D. E. (2012). Evaluating the economic response to japan's earthquake. Policy Discussion Paper, 12, P003.

Silva, J. S., \& Tenreyro, S. (2006). The log of gravity. The Review of Economics and Statistics, 88(4), 641658.

Silva, J. S., \& Tenreyro, S. (2010). On the existence of the maximum likelihood estimates in Poisson regression. Economics Letters, 107(2), 310-312.

Solis, M., \& Urata, S. (2018). Abenomics and japan's trade policy in a new era. Asian Economic Policy Review, 13(1), 106-123.

Tinbergen, J. (1962). Shaping the world economy; suggestions for an international economic policy.

Wooldridge, J. M. (2016). Introductory econometrics: A modern approach / Jeffrey M. Wooldridge, Michigan state university. Australia: Cengage Learning.

Yotov, Y. V., Piermartini, R., Monteiro, J., \& Larch, M. (2016). An advanced guide to trading policy analysis: The structural gravity model World Trade Organization Geneva. Retrieved from https://vi.unctad.org/tpa/

Yotov, Y. V., Piermartini, R., Monteiro, J., \& Larch, M. (2017). Partial equilibrium trade policy analysis with structural gravity. New York: United Nations. doi:10.18356/b7cecad8-en 